\title{
Central-force decomposition of spline-based modified embedded atom method potential
}

Article in Modelling and Simulation in Materials Science and Engineering · September 2016

DOI: $10.1088 / 0965-0393 / 24 / 7 / 075003$

CITATIONS

0

3 authors:

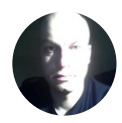

Szymon Winczewski

Gdansk University of Technology

8 PUBLICATIONS 38 CITATIONS

SEE PROFILE

Jarosław Rybicki

Gdansk University of Technology

78 PUBLICATIONS 353 CITATIONS

SEE PROFILE
Jacek Dziedzic

University of Southampton

22 PUBLICATIONS 79 CITATIONS

SEE PROFILE 
(C) 2016. This manuscript version is made available under the CC-BY-NC-ND 4.0 license:

http://creativecommons.org/licenses/by-nc-nd/4.0/

The formal publication can be found here:

http://dx.doi.org/10.1088/0965-0393/24/7/075003

\title{
Central-force decomposition of spline-based modified embedded atom method potential
}

\author{
S Winczewski ${ }^{1}$, J Dziedzic ${ }^{1,2}$ and J Rybicki ${ }^{1,3}$ \\ ${ }^{1}$ Faculty of Applied Physics and Mathematics, Gdansk University of \\ Technology, Narutowicza 11/12, 80-233 Gdańsk, Poland \\ ${ }^{2}$ School of Chemistry, University of Southampton, Highfield, Southampton \\ SO17 1BJ, UK \\ 3 TASK Computer Centre, Gdansk University of Technology, Narutowicza \\ 11/12, 80-233 Gdańsk, Poland \\ E-mail: wisnia@kdm.task.gda.pl
}

\begin{abstract}
Central-force decompositions are fundamental to the calculation of stress fields in atomic systems by means of Hardy stress. We derive expressions for a central-force decomposition of the spline-based modified embedded atom method (s-MEAM) potential. The expressions are subsequently simplified to a form that can be readily used in molecular-dynamics simulations, enabling the calculation of the spatial distribution of stress in systems treated with this novel class of empirical potentials. We briefly discuss the properties of the obtained decomposition and highlight further computational techniques that can be expected to benefit from the results of this work. To demonstrate the practicability of the derived expressions, we apply them to calculate stress fields due to an edge dislocation in bcc Mo, comparing their predictions to those of linear elasticity theory.

Keywords: central-force decomposition, atomic stress, embedded atom method, molecular dynamics, empirical potentials
\end{abstract}

PACS numbers: $02.70 . \mathrm{Ns}, 62.20 .-\mathrm{x}, 34.20 . \mathrm{Cf}$

Submitted to: Modelling Simul. Mater. Sci. Eng. 


\section{Introduction}

Molecular-dynamics (MD) simulations constitute an indispensable tool for the computational theorist. By presenting a spatial-temporal picture of the behaviour of matter at the atomic scale they can elucidate aspects of physical processes and phenomena that are often inaccessible to experimental study.

The credibility of results obtained from any atomistic simulation depends first and foremost on the quality of the model employed to describe interatomic interactions. The last two decades witnessed significant improvements in empirical descriptions of the condensed phase, with a number of novel potentials developed for covalent [1-13], ionic $[14,15]$, and metallic systems [16-22]. Recently developed potentials are able to satisfactorily describe the properties of wide classes of materials, with quantitative agreement with experiment often attained or within sight.

For metals and their alloys the embedded atom method [23] (EAM) represents the state of the art. EAM-type potentials have been developed for the majority of metallic elements $[16,18,21,24,25]$, with as many as several potentials or parameterisations available for a number of metals (e.g.: $\mathrm{Al}$ and $\mathrm{Ni}[16,21,24]$; $\mathrm{Ag}, \mathrm{Au}, \mathrm{Cu}$ and Pt $[18,21,24])$. A range of generalisations and extensions of EAM have been proposed and studied - the modified embedded atom method [26] (MEAM), first nearestneighbour modified embedded atom method [27] (1NN-MEAM), second nearestneighbour modified embeded atom method [17] (2NN-MEAM), angular-dependent embedded atom method [20] (A-EAM) and spline-based modified embedded atom method [11] (s-MEAM). All the above-mentioned improvements employ a more general form of the description with the aim of correctly capturing the nature of the interactions for configurations that are far from the equilibrium structure. Successes have recently been reported even for problematic systems, such as $\mathrm{Zr}$ or $\mathrm{Ti}$ [28]. Newly-developed potentials often offer improved transferability, occasionally aiming to reproduce wide subsets of phase diagrams of binary systems [29]. Of the generalisations listed above the s-MEAM approach deserves particular attention.

The spline-based modified embedded atom method (s-MEAM) was proposed in 2000 by Lenosky et al [11] in order to describe the interactions in silicon. The starting point is the MEAM formalism [27], with the traditionally used functional forms subsequently replaced with cubic spline functions. Furthermore, the description of the electronic density has been augmented with angular terms of very general nature, obviating the need to decide a priori on a particular character of angular dependence. Lenosky et al [11] demonstrate that their potential describes well the energetics of a wide range of configurations for Si (including the dimer, fcc and hcp) and is able to reproduce structural and elastic properties of a number of phases of bulk silicon.

More recently it was shown [22] that - when thoroughly parameterised to reproduce properties obtained from $a b$ initio calculations - the s-MEAM approach can yield a remarkably predictive and transferable potential. The potential developed in 2012 by Park et al [22] offers a very good description of a number of properties of molybdenum, including structural (lattice constants, energetics of stable and metastable phases), elastic (elastic constants, phonon dispersion curves), and thermodynamic properties (coefficients of thermal expansion, compressibility). Moreover, the properties of defected Mo are also well described, both for point and line defects, with the latter of particular interest in studies of plasticity [30]. Through a comparison with ab initio calculations Park et al were able to demonstrate that the proposed potential is able to reasonably well describe fundamental properties 
of dislocations, such as dislocation energy or Peierls stress, and other descriptors of plastic behaviour of materials, e.g. shear strength and stacking fault energies.

Currently s-MEAM parameterisations are available only for Mo [22], Ti [31] and $\mathrm{Si}[11,32]$. The parameterisation for Ti has been particularly successful, with the ability to describe complex martensitic phase transformations between the $\alpha, \beta$ and $\omega$ phases of titanium. At the same time its predictions for elastic constants, phonon frequencies, surface energies and defect formation energies are in very good agreement with results obtained at the density functional theory (DFT) level of theory. With the successes of $[11,22,31,32]$ in mind, we anticipate an increase in the number of available parameterisations of s-MEAM in the near future.

More often than not, the full characterisation of a simulated material requires the determination of its stress distribution. This is of particular interest in the field of mechanics of materials, where the ability to describe the behaviour of matter at the picosecond-nanometre-scale in the familiar terms used in continuum theory is desirable for the elucidation of the effect of microstructure on the macroscopic properties of the material.

Stress is a well-defined quantity in continuum mechanics. The task of formulating a suitable, physically sound atomic-scale analogue is difficult, which is evidenced by the fact that it took over a hundred years for consesus to be reached in the literature.

Clausius and Maxwell, whose works [33-35] presented the virial theorem, can be regarded as the first to tackle the microscopic definition of stress. The concept of virial stress was later developed by Tsai [36], who generalised its definition to the case of finite temperature. Owing to its simple form and ease of calculation, virial stress became a commonly used measure of macroscopic stress in atomistic simulations.

The need to describe the spatial distribution of stress led to the formulation of measures of local stress that were based on virial stress. In such approaches local stress is usually calculated at atomic sites, taking into account the contributions to the virial associated with the atom in question [37] (Basinski-Duesberry-Taylor (BDT) stress). Alternatively, local stress at a point can be expressed in terms of contributions to the virial that arise from an averaging volume encompassing the point in question [38] (Lutsko stress). The validity of the above virial-based classes of approaches has since been questioned. For instance, it has been shown [39] that they do not correspond to the true (Cauchy) stress, or any other mechanical stress. Limitations in their application to non-homogeneous and non-equilibrium systems have also been highlighted [40].

Another line of attack on the problem of determining local stress was taken in the 1950s by Irving and Kirkwood, who described [41] a formalism for determining fields of certain quantities, among these the stress tensor, for any given distribution of particles. The IK approach is based on non-equilibrium classical statistical mechanics and, by construction, can be applied to non-homogeneous and non-equilibrium systems. The approach is, however, plagued by practical difficulties which mostly result from the expansions into a series of Dirac delta distributions that are present in the final expressions.

The Irving-Kirkwood approach was later generalised by Noll [42], who proved two significant lemmata which could be used to rid the IK procedure of the incumbrance of Dirac delta series. Despite the fact that the expressions obtained by Noll were significantly more compact, the modified approach was still far from straightforward, with the difficulties mostly a consequence of its purely stochastic nature.

A breakthrough materialised in the works of Hardy [43] and Murdoch [44], who 
independently arrived at a new formulation, one that worked around the mathematical difficulties of the IK procedure. Hardy and Murdoch noted that experimental measurements reflect spatial-temporal averages of molecular behaviour. Hardy [43] replaced the Dirac delta distributions arising in the Irvin-Kirkwood approach with regular functions, termed kernel functions, which served to perform spatial averaging. Murdoch [44] introduced spatial and temporal averaging to the IK procedure, which was another way of circumventing the use of Dirac delta distributions and obviated the need to calculate ensemble averages.

The approach of $[43,44]$ (termed the Murdoch-Hardy procedure, with the corresponding quantity known as Hardy stress) was later further generalised [45-54]. Owing to its many advantages, Hardy stress has since found widespread use in atomistic simulations [55-64].

Other approaches to the calculation of local stress have been proposed over time [36-38, 40,65], with the definition itself often an object of debate [40,48,50,51,53-55]. The features of a number of measures of local stress were examined in detail by Admal and Tadmor $[53,54]$, who showed through numerical experiments [53] that of the three most commonly used approaches, Hardy stress is the most accurate approach, with the most favourable convergence with respect to the averaging volume.

It is worth pointing out, however, that the spectrum of application of Hardy stress is still limited in practice, which is a consequence of the assumptions regarding the form of energies and forces imposed by the framework of Murdoch-Hardy's formalism, viz.:

(i) the potential energy of interactions $E_{\text {pot }}$ is a function of only the interatomic distances, i.e. it can be expressed as

$$
E_{\text {pot }}=E_{\text {pot }}\left(r_{12}, r_{13}, \ldots, r_{1 N}, r_{23}, r_{24}, \ldots, r_{2 N}, \ldots, r_{N-1, N}\right),
$$

(ii) the total force acting on atom $i$ can be written as

$$
\mathbf{F}_{i}=\sum_{j \neq i} \mathbf{F}_{i j}
$$

where $\mathbf{F}_{i j}$ is the force with which atom $j$ acts on atom $i$. The forces must satisfy the strong law of action and reaction, i.e. $\mathbf{F}_{i j}=-\mathbf{F}_{j i}$ and the force $\mathbf{F}_{i j}$ must be a central force, i.e. the vector $\mathbf{F}_{i j}$ must be parallel to the vector $\mathbf{r}_{i j}$ connecting atoms $i$ and $j$.

A method for decomposing total forces that satisfies the conditions stated in the second point above is termed a central force decomposition (CFD). This is a natural, and, as shown by Admal and Tadmor [53], the most justifiable decomposition, because it provides a stress tensor which is symmetric and which also satisfies the balance of linear and angular momentum.

While all pair potentials satisfy both (1) and (2), it is not immediately obvious whether the above conditions, and (2) in particular, are satisfied in the case of more involved potentials, particularly for many-body potentials. For this reason, Hardy stress has so far been employed in calculations using pair potentials [58,61], or EAM potentials that use a spherically symmetric form of the electronic density $[55,57,59,60,62]$, for which CFD expressions are known. The possibility of applying Hardy stress to three-body potentials has also been discussed [56]. Only recently applications of Hardy stress to systems described by four-body potentials have been reported $[63,64]$. To our best knowledge, there are no examples in the literature of 
the use of Hardy stress in systems described by many-body potentials more involved than the original EAM potential.

Finding a suitable central force decomposition for the potential of choice is a prerequisite for using Hardy's formalism. In [53] it was shown that for any multi-body potential with a continuously differentiable extension, the force that acts on a particle can always be expressed as a sum of central forces. In this work, building on the observations of Admal and Tadmor [53], we present expressions for the central force decomposition for potentials of the s-MEAM class [11], the popularity of which we expect to rise significantly in the near future.

This paper is organised as follows. In Section 2 we recap the s-MEAM approach and the definition of Hardy stress, following with crucial observations regarding CFD. In Section 3 we present the main result of this work, which is a step-by-step derivation of the expressions for CFD for s-MEAM potentials. Section 4 is devoted to a discussion of the particular features of the obtained CFD expressions. We demonstrate an example application of the derived expressions, along with a discussion of obtained results in Section 5. We conclude with a summary.

\section{Formalism}

\subsection{Notation}

The following notation will be used throughout this work. We will use $\mathbf{r}_{i}$ to denote the position vector of particle $i$, i.e. $\mathbf{r}_{i}=\left(x_{i}, y_{i}, z_{i}\right)$. A vector joining particle $i$ with partcile $j$ will be denoted with $\mathbf{r}_{i j}=\mathbf{r}_{j}-\mathbf{r}_{i}$, and its magnitude with $r_{i j}=\left|\mathbf{r}_{i j}\right|$. The valence angle between bonds $\mathbf{r}_{i j}$ and $\mathbf{r}_{i k}$ will be denoted with $\theta_{j i k}$, with

$$
\cos \theta_{j i k}=\frac{\mathbf{r}_{i j} \cdot \mathbf{r}_{i k}}{r_{i j} r_{i k}} .
$$

The nearest-neighbour cutoff radius will be denoted with $r_{\mathrm{c}}$, and by first-nearest neighbours of atom $i$ we will understand all atoms $j \neq i$, for which $r_{i j} \leq r_{\mathrm{c}}$. The set of first-nearest neighbours of atom $i$ will be denoted with $F(i)$. By second-nearest neighbours of atom $i$ we will understand all atoms $j$, for which $r_{\mathrm{c}}<r_{i j} \leq 2 r_{\mathrm{c}}$. The set of second-nearest neighbours of atom $i$ will be denoted with $S(i)$. The union of the two above sets will be denoted with $B(i)=F(i) \cup S(i)$. For the sake of brevity we shall use

$$
h^{\prime}\left(x_{0}\right)=\left.\frac{\mathrm{d} h(x)}{\mathrm{d} x}\right|_{x=x_{0}}
$$

to denote the first derivative of $h(x)$ evaluated at $x=x_{0}$.

\subsection{The s-MEAM potential}

In the s-MEAM model [11] the potential energy of a system of $N$ particles is given by

$$
E_{\mathrm{pot}}=\frac{1}{2} \sum_{i} \sum_{j \neq i} \phi\left(r_{i j}\right)+\sum_{i} U\left(n_{i}\right) .
$$

The first term $(\phi(r))$ describes the pairwise repulsion of the cores, while the second term represents the cohesion energy resulting from embedding positively-charged ionic 
cores inside the electronic density, described by $U(n)$. The quantity $n_{i}$ is the electronic density at the position of particle $i$, and is given by

$$
n_{i}=\sum_{j \neq i} \rho\left(r_{i j}\right)+\frac{1}{2} \sum_{j \neq i} \sum_{\substack{k \neq i \\ k \neq j}} f\left(r_{i j}\right) f\left(r_{i k}\right) g\left(\cos \theta_{j i k}\right) .
$$

The function $\rho(r)$ describes the terms in $n_{i}$ that have spherical symmetry, with the second term in (6) grouping the terms with no such symmetry.

The general form of the potential given by (5) and (6) is identical to that proposed by Baskes for silicon [27]. The Stillinger-Weber potential [66] constitutes a special case of this form (with $U(x)=x$ and $\rho=0$ ). Numerous EAM potentials [23] are also a special case (with $f=0$ or $g=0$ ).

Within the s-MEAM framework all of the functions $\phi(r), U(n), \rho(r), f(r)$ and $g(\cos \theta)$ are represented as (usually cubic) spline functions. The functions $\phi(r), \rho(r)$ and $f(r)$ additionally serve as cutoff functions, i.e. they smoothly vanish beyond a specified cutoff. While in principle the cutoff for each function can be different, one can always specify a suitably large cutoff radius $r_{\mathrm{c}}$, for which all the functions vanish.

The s-MEAM approach is characterised by high computational efficiency. This is a consequence of the use of spline functions, which can be efficently evaluated. Hennig et al [31] observed that the use of s-MEAM is only about twice as computationally intensive as standard EAM [23]. An efficient evaluation technique exists for s-MEAM potentials [67], and its implementation can be found in popular MD codes, e.g. in LAMMPS [68]. The use of spline functions also facilitates parameterisation, and not using a predetermined functional form avoids imposing any particular functional character on the potential.

\subsection{Hardy stress}

Hardy stress at a point $\mathbf{x}$ is defined as a sum of two contributions [64]

$$
\boldsymbol{\sigma}(\mathbf{x})=\boldsymbol{\sigma}^{\mathrm{K}}(\mathbf{x})+\boldsymbol{\sigma}^{\mathrm{V}}(\mathbf{x}),
$$

with the kinetic term $\sigma^{\mathrm{K}}(\mathbf{x})$ given as

$$
\boldsymbol{\sigma}^{\mathrm{K}}(\mathbf{x})=-\sum_{i} w\left(\mathbf{x} ; \mathbf{r}_{i}-\mathbf{x}\right) m_{i} \mathbf{v}_{i} \otimes \mathbf{v}_{i},
$$

and the potential term $\boldsymbol{\sigma}^{\mathrm{V}}(\mathbf{x})$ as

$$
\boldsymbol{\sigma}^{\mathrm{V}}(\mathbf{x})=\frac{1}{2} \sum_{i} \sum_{j \neq i} B\left(\mathbf{x} ; \mathbf{r}_{i}, \mathbf{r}_{j}\right) \mathbf{F}_{i j} \otimes \mathbf{r}_{i j},
$$

with $m_{i}$ denoting the mass of particle $i, \mathbf{v}_{i}$ - its velocity, and $\mathbf{F}_{i j}$ - the (central) force of interaction between particles $i$ and $j$. The symbol $\otimes$ denotes a dyadic product.

The kinetic term $\boldsymbol{\sigma}^{\mathrm{K}}(\mathbf{x})$ is due to the flux of momentum associated with the vibrational internal energy of the system. The potential term $\boldsymbol{\sigma}^{\mathrm{V}}(\mathbf{x})$ arises from the internal forces between the particles.

In some formulations [63,64], most often for systems in equilibrium, time averaging is employed for both of the above terms. In the absence of time averaging the instantaneous stress tensor is obtained.

The function $w(\mathbf{x}, \mathbf{y})$ (variously termed the kernel function [50,53], the localisation function $[48,51,56,57,64]$, or the weighting function $[48,63]$ ) is involved in spatial averaging. The kernel function is chosen to have a compact support, its domain $\Omega_{\mathrm{x}}$ is 
centered at the point $\mathbf{x}$, defining a region around it over which averaging is performed. The function serves to couple the atomic and continuum descriptions: it smears out the discrete nature of matter by assigning weights to the contributions to $\boldsymbol{\sigma}(\mathbf{x})$ due to particles at $\mathbf{y} \in \Omega_{\mathbf{x}}$, with closer particles assigned larger weights.

The kernel function is also present in the definition of the bond function $B\left(\mathbf{x} ; \mathbf{r}_{i}, \mathbf{r}_{j}\right)$ that appears in (9). The bond function is defined as

$$
B\left(\mathbf{x} ; \mathbf{r}_{i}, \mathbf{r}_{j}\right)=\int_{0}^{1} \mathrm{~d} s w\left(\mathbf{x} ;(1-s) \mathbf{r}_{i}+s \mathbf{r}_{j}\right) .
$$

The calculation of $\boldsymbol{\sigma}^{\mathrm{V}}(\mathbf{x})$ involves integrating $w\left(\mathbf{x}, \mathbf{r}_{i}, \mathbf{r}_{j}\right)$ along the segment joining particle $i$ (corresponding to $s=0$ ) with particle $j$ (corresponding to $s=1$ ). The potential term $\boldsymbol{\sigma}^{\mathrm{V}}(\mathbf{x})$ thus expresses the spatial average of interactions that span the volume $\Omega_{\mathbf{x}}$. The contribution $\mathbf{F}_{i j}$ to $\boldsymbol{\sigma}^{\mathrm{V}}(\mathbf{x})$ depends on the length of the segment joining $\mathbf{r}_{i}$ and $\mathbf{r}_{j}$ and its distance to (and orientation with respect to) $\Omega_{\mathbf{x}}$.

The function $w(\mathbf{x}, \mathbf{y})$ is chosen such that it is i) smooth and non-negative, ii) normalised:

$$
\int_{\Omega_{\mathbf{x}}} \mathrm{d} \mathbf{y} w(\mathbf{x} ; \mathbf{y})=1
$$

iii) has a maximum at $\mathbf{y}=\mathbf{x}$ and decreases with the distance $|\mathbf{x}-\mathbf{y}|$. Since the function $w(\mathbf{x}, \mathbf{y})$ is used for spatial averaging, it should be chosen such that its domain $\Omega_{\mathbf{x}}$ is large enough to prevent individual atoms from skewing the stress, and simultaneously small enough to observe spatial variations of the stress on physically relevant length scales. The functional forms of $w(\mathbf{x}, \mathbf{y})$ used in practice include: a Gaussian function [50,53], trilinear weight functions [64], quartic splines [53], quartic polynomials [57] and other, more complex forms [63].

\subsection{Central-force decomposition}

Hardy stress is based around the concept of a central force $\mathbf{F}_{i j}$ acting along the vector $\mathbf{r}_{i j}$ connecting particles $i$ and $j$. Admal and Tadmor showed [53] that if $\mathbf{F}_{i j}$ is a central force then the pointwise stress tensor $\boldsymbol{\sigma}(\mathbf{x})$ defined by (7)-(9) is symmetric and satisfies a balance of linear and angular momentum. For pair potentials the total force $\mathbf{F}_{i}$ can be immediately expressed in the form necessitated by (2), satisfying $\mathbf{F}_{i j}=-\mathbf{F}_{j i}$ and $\mathbf{F}_{i j} \| \mathbf{r}_{i j}$. In the case of many-body potentials expressing total forces in terms of central forces is not trivial, and the expressions are known as the central-force decomposition (CFD).

As shown by Admal et al $[53,54]$, the total force acting on particle $i$ is guaranteed to be decomposable into central forces if the interatomic potential energy, $E_{\text {pot }}$, defined on a shape space

$$
\mathcal{S}:=\left\{r_{12}, r_{13}, \ldots, r_{1 N}, r_{23}, r_{24}, \ldots, r_{2 N}, \ldots, r_{N-1, N}\right\},
$$

is continuously differentiable. Furthermore [53], a central-force decomposition can be obtained by finding the derivative of the potential $E_{\text {pot }}$ with respect to the interatomic distance $r_{i j}$. The application of the chain rule lets us express $\mathbf{F}_{i}$ as

$$
\mathbf{F}_{i}=-\frac{\partial E_{\mathrm{pot}}}{\partial \mathbf{r}_{i}}=\sum_{j \neq i} \frac{\partial E_{\mathrm{pot}}}{\partial r_{i j}} \frac{\mathbf{r}_{i j}}{r_{i j}},
$$

and (by comparison with (2)) thus

$$
\mathbf{F}_{i j}=\frac{\partial E_{\mathrm{pot}}}{\partial r_{i j}} \frac{\mathbf{r}_{i j}}{r_{i j}}
$$


It is easy to verify that the force $\mathbf{F}_{i j}$ given by (14) satisfies both conditions of the strong law of action and reaction: $\mathbf{F}_{i j}=-\mathbf{F}_{j i}$ and $\mathbf{F}_{i j} \| \mathbf{r}_{i j}$. Following Admal et al [53] we stress that it is not a requirement for $\mathbf{F}_{i j}$ to depend only on the positions of particles $i$ and $j$. In general, $\mathbf{F}_{i j}$ can depend on the positions of any (even all) particles in the system, being a function of up to $N(N-1) / 2$ interatomic distances. From the fact that the force $\mathbf{F}_{i j}$ (a derivative $\partial E_{\text {pot }} / \partial r_{i j}$ ) is not expressible as a function of only $r_{i j}$ it does not follow that the potential is not decomposable into central forces, as is often intuitively (but incorrectly) expected. Finally, we reiterate Admal's statement [53] that for a system of $N \geq 5$ particles there may, in general, exist distinct methods of decomposing a potential into central forces, due to the nonuniqueness of the interatomic potential extension.

\section{Central-force decomposition of s-MEAM potential}

In this section we derive the expression for the first derivative of an s-MEAM-type potential energy expression with respect to the interatomic distance, i.e. $\partial E_{\mathrm{pot}} / \partial r_{\alpha \beta}$. The central-force decomposition can then be obtained by calculating the central force $\mathbf{F}_{\alpha \beta}$ according to (13) and (14).

By applying the chain rule in the differentiation of (5), we obtain

$$
\begin{aligned}
\frac{\partial E_{\mathrm{pot}}}{\partial r_{\alpha \beta}} & =\frac{1}{2} \sum_{i} \sum_{j \neq i} \frac{\partial}{\partial r_{\alpha \beta}} \phi\left(r_{i j}\right)+\sum_{i} \frac{\partial}{\partial r_{\alpha \beta}} U\left(n_{i}\right) \\
& =\frac{1}{2} \sum_{i} \sum_{j \neq i} \phi^{\prime}\left(r_{i j}\right) \frac{\partial r_{i j}}{\partial r_{\alpha \beta}}+\sum_{i} U^{\prime}\left(n_{i}\right) \frac{\partial n_{i}}{\partial r_{\alpha \beta}} .
\end{aligned}
$$

The derivative $\partial r_{i j} / \partial r_{\alpha \beta}$ can be written as

$$
\frac{\partial r_{i j}}{\partial r_{\alpha \beta}}=\delta_{\alpha i} \delta_{\beta j}+\delta_{\alpha j} \delta_{\beta i}
$$

where $\delta$ is the Kronecker delta. By inserting (16) in the first term of (15), we obtain

$$
\frac{\partial E_{\mathrm{pot}}}{\partial r_{\alpha \beta}}=\phi^{\prime}\left(r_{\alpha \beta}\right)+\sum_{i} U^{\prime}\left(n_{i}\right) \frac{\partial n_{i}}{\partial r_{\alpha \beta}} .
$$

The above makes use of $r_{\alpha \beta}=r_{\beta \alpha}$ and $\phi^{\prime}\left(r_{\alpha \beta}\right)=\phi^{\prime}\left(r_{\beta \alpha}\right)$. The more elaborate derivation for $\partial n_{i} / \partial r_{\alpha \beta}$ will be shown separately.

By acting the operator $\partial / \partial r_{\alpha \beta}$ on (6) we obtain

$\frac{\partial n_{i}}{\partial r_{\alpha \beta}}=\sum_{j \neq i} \rho^{\prime}\left(r_{i j}\right) \frac{\partial r_{i j}}{\partial r_{\alpha \beta}}+\frac{1}{2} \sum_{\substack{j \neq i \\ j \neq i \\ k \neq j}} \frac{\partial}{\partial r_{\alpha \beta}}\left[f\left(r_{i j}\right) f\left(r_{i k}\right) g\left(\cos \theta_{j i k}\right)\right]$.

The application of the product rule leads to

$$
\begin{aligned}
\frac{\partial}{\partial r_{\alpha \beta}}\left[f\left(r_{i j}\right) f\left(r_{i k}\right) g\left(\cos \theta_{j i k}\right)\right]= & \left(f^{\prime}\left(r_{i j}\right) \frac{\partial r_{i j}}{\partial r_{\alpha \beta}}\right) f\left(r_{i k}\right) g\left(\cos \theta_{j i k}\right) \\
& +f\left(r_{i j}\right)\left(f^{\prime}\left(r_{i k}\right) \frac{\partial r_{i k}}{\partial r_{\alpha \beta}}\right) g\left(\cos \theta_{j i k}\right) \\
& +f\left(r_{i j}\right) f\left(r_{i k}\right)\left(g^{\prime}\left(\cos \theta_{j i k}\right) \frac{\partial \cos \theta_{j i k}}{\partial r_{\alpha \beta}}\right) .
\end{aligned}
$$


The expressions (16) and (18) allow us to recast the second term of (17) as

$$
\begin{aligned}
\sum_{i} U^{\prime}\left(n_{i}\right) \frac{\partial n_{i}}{\partial r_{\alpha \beta}}= & \sum_{i} U^{\prime}\left(n_{i}\right) \\
& \times\left\{\sum_{j \neq i} \rho^{\prime}\left(r_{i j}\right) \frac{\partial r_{i j}}{\partial r_{\alpha \beta}}+\frac{1}{2} \sum_{\substack { j \neq i \\
\begin{subarray}{c}{k \neq i \\
k \neq j{ j \neq i \\
\begin{subarray} { c } { k \neq i \\
k \neq j } }\end{subarray}} \frac{\partial}{\partial r_{\alpha \beta}}\left[f\left(r_{i j}\right) f\left(r_{i k}\right) g\left(\cos \theta_{j i k}\right)\right]\right\} \\
= & \sum_{i} \sum_{j \neq i} U^{\prime}\left(n_{i}\right) \rho^{\prime}\left(r_{i j}\right)\left(\delta_{\alpha i} \delta_{\beta j}+\delta_{\alpha j} \delta_{\beta i}\right) \\
& +\frac{1}{2} \sum_{i} \sum_{j \neq i} \sum_{\substack{k \neq i \\
k \neq j}} U^{\prime}\left(n_{i}\right) \frac{\partial}{\partial r_{\alpha \beta}}\left[f\left(r_{i j}\right) f\left(r_{i k}\right) g\left(\cos \theta_{j i k}\right)\right] \\
= & U^{\prime}\left(n_{\alpha}\right) \rho^{\prime}\left(r_{\alpha \beta}\right)+U^{\prime}\left(n_{\beta}\right) \rho^{\prime}\left(r_{\beta \alpha}\right)+Z
\end{aligned}
$$

where we have introduced the symbol $Z$ for the sake of compactness of notation. Referring to (19) we can express $Z$ as

$$
\begin{aligned}
& Z=\frac{1}{2} \sum_{i} \sum_{j \neq i} \sum_{\substack{k \neq i \\
k \neq j}} U^{\prime}\left(n_{i}\right)\left(f^{\prime}\left(r_{i j}\right) \frac{\partial r_{i j}}{\partial r_{\alpha \beta}}\right) f\left(r_{i k}\right) g\left(\cos \theta_{j i k}\right) \\
& +\frac{1}{2} \sum_{i} \sum_{j \neq i} \sum_{\substack{k \neq i \\
k \neq j}} U^{\prime}\left(n_{i}\right) f\left(r_{i j}\right)\left(f^{\prime}\left(r_{i k}\right) \frac{\partial r_{i k}}{\partial r_{\alpha \beta}}\right) g\left(\cos \theta_{j i k}\right) \\
& +\frac{1}{2} \sum_{i} \sum_{j \neq i} \sum_{\substack{k \neq i \\
k \neq j}} U^{\prime}\left(n_{i}\right) f\left(r_{i j}\right) f\left(r_{i k}\right)\left(g^{\prime}\left(\cos \theta_{j i k}\right) \frac{\partial \cos \theta_{j i k}}{\partial r_{\alpha \beta}}\right) \\
& =Z_{1}+Z_{2}+Z_{3} \text {, }
\end{aligned}
$$

where we have introduced $Z_{1}, Z_{2}$, and $Z_{3}$ for the sake of brevity.

The application of (16) lets us write $Z_{1}$ as

$$
Z_{1}=\frac{1}{2} \sum_{i} \sum_{j \neq i} \sum_{\substack{k \neq i \\ k \neq j}}\left[\delta_{\alpha i} \delta_{\beta j}+\delta_{\alpha j} \delta_{\beta i}\right] U^{\prime}\left(n_{i}\right) f^{\prime}\left(r_{i j}\right) f\left(r_{i k}\right) g\left(\cos \theta_{j i k}\right),
$$

which, owing to the Kronecker deltas, is reduced to

$$
Z_{1}=U^{\prime}\left(n_{\alpha}\right) f^{\prime}\left(r_{\alpha \beta}\right) \sum_{\substack{i \neq \alpha \\ i \neq \beta}} f\left(r_{\alpha i}\right) g\left(\cos \theta_{\beta \alpha i}\right) .
$$

Similarly, we obtain

$$
Z_{2}=U^{\prime}\left(n_{\beta}\right) f^{\prime}\left(r_{\beta \alpha}\right) \sum_{\substack{i \neq \beta \\ i \neq \alpha}} f\left(r_{\beta i}\right) g\left(\cos \theta_{\alpha \beta i}\right) .
$$

In both cases we renamed dummy indices, replacing $k$ with $i$.

The term $Z_{3}$ occuring in (21) is more involved, as it includes the derivative $\partial \cos \theta_{j i k} / \partial r_{\alpha \beta}$. As the angle $\theta_{j i k}$ can depend on $r_{\alpha \beta}$ in six ways (through $\theta_{\beta \alpha i}$, $\theta_{\alpha \beta i}, \theta_{i \alpha \beta}$ and $\theta_{i \beta \alpha}$; through $\left.\theta_{\alpha i \beta}, \theta_{\beta i \alpha}\right)$, the derivative $\partial \cos \theta_{j i k} / \partial r_{\alpha \beta}$ can be written as

$$
\frac{\partial \cos \theta_{j i k}}{\partial r_{\alpha \beta}}=\left(\delta_{\alpha i} \delta_{\beta j}+\delta_{\alpha j} \delta_{\beta i}\right) \frac{\partial \cos \theta_{j i k}}{\partial r_{i j}}
$$




$$
\begin{aligned}
& +\left(\delta_{\alpha i} \delta_{\beta k}+\delta_{\alpha k} \delta_{\beta i}\right) \frac{\partial \cos \theta_{j i k}}{\partial r_{i k}} \\
& +\left(\delta_{\alpha j} \delta_{\beta k}+\delta_{\alpha k} \delta_{\beta j}\right) \frac{\partial \cos \theta_{j i k}}{\partial r_{j k}} .
\end{aligned}
$$

We have used the fact that $r_{i j}=r_{j i}$, and thus $\partial \cos \theta_{j i k} / \partial r_{i j}=\partial \cos \theta_{j i k} / \partial r_{j i}$.

In order to determine the three derivatives in (25) we are going to employ the cosine rule

$$
\cos \theta_{j i k}=\frac{r_{i j}^{2}+r_{i k}^{2}-r_{j k}^{2}}{2 r_{i j} r_{i k}}
$$

along with its derivatives

$$
\begin{aligned}
& \frac{\partial \cos \theta_{j i k}}{\partial r_{i j}}=\frac{\cos \theta_{j i k}}{r_{i j}}-\frac{r_{i k}^{2}-r_{j k}^{2}}{r_{i k}} \frac{1}{r_{i j}^{2}}, \\
& \frac{\partial \cos \theta_{j i k}}{\partial r_{i k}}=\frac{\cos \theta_{j i k}}{r_{i k}}-\frac{r_{i j}^{2}-r_{j k}^{2}}{r_{i j}} \frac{1}{r_{i k}^{2}},
\end{aligned}
$$

and

$$
\frac{\partial \cos \theta_{j i k}}{\partial r_{j k}}=-\frac{r_{j k}}{r_{i j} r_{i k}} .
$$

Combining (27), (28), and (29) with (25), we obtain

$$
\begin{aligned}
\frac{\partial \cos \theta_{j i k}}{\partial r_{\alpha \beta}}= & \left(\delta_{\alpha i} \delta_{\beta j}+\delta_{\alpha j} \delta_{\beta i}\right)\left[\frac{\cos \theta_{j i k}}{r_{i j}}-\frac{r_{i k}^{2}-r_{j k}^{2}}{r_{i k}} \frac{1}{r_{i j}^{2}}\right] \\
& +\left(\delta_{\alpha i} \delta_{\beta k}+\delta_{\alpha k} \delta_{\beta i}\right)\left[\frac{\cos \theta_{j i k}}{r_{i k}}-\frac{r_{i j}^{2}-r_{j k}^{2}}{r_{i j}} \frac{1}{r_{i k}^{2}}\right] \\
& -\left(\delta_{\alpha j} \delta_{\beta k}+\delta_{\alpha k} \delta_{\beta j}\right) \frac{r_{j k}}{r_{i j} r_{i k}}
\end{aligned}
$$

Having expanded all the products in (30), we can express the quantity $Z_{3}$ that appears in (21) as a sum of ten terms, viz.:

$$
Z_{3}=Z_{3,1}+Z_{3,2}+\ldots+Z_{3,10}
$$

where

$$
\begin{gathered}
Z_{3,1}=\frac{1}{2} \sum_{i} \sum_{j \neq i} \sum_{\substack{k \neq i \\
k \neq j}} U^{\prime}\left(n_{i}\right) f\left(r_{i j}\right) f\left(r_{i k}\right) g^{\prime}\left(\cos \theta_{j i k}\right) \delta_{\alpha i} \delta_{\beta j} \frac{\cos \theta_{j i k}}{r_{i j}} \\
=\frac{1}{2} U^{\prime}\left(n_{\alpha}\right) \frac{f\left(r_{\alpha \beta}\right)}{r_{\alpha \beta}} \sum_{\substack{i \neq \alpha \\
i \neq \beta}} f\left(r_{\alpha i}\right) g^{\prime}\left(\cos \theta_{\beta \alpha i}\right) \cos \theta_{\beta \alpha i}, \\
Z_{3,2}=\frac{1}{2} \sum_{i} \sum_{j \neq i} \sum_{\substack{k \neq i \\
k \neq j}} U^{\prime}\left(n_{i}\right) f\left(r_{i j}\right) f\left(r_{i k}\right) g^{\prime}\left(\cos \theta_{j i k}\right) \delta_{\alpha i} \delta_{\beta j}\left(-\frac{r_{i k}^{2}-r_{j k}^{2}}{r_{i k}} \frac{1}{r_{i j}^{2}}\right) \\
=-\frac{1}{2} U^{\prime}\left(n_{\alpha}\right) \frac{f\left(r_{\alpha \beta}\right)}{r_{\alpha \beta}^{2}} \sum_{\substack{i \neq \alpha \\
i \neq \beta}} f\left(r_{\alpha i}\right) g^{\prime}\left(\cos \theta_{\beta \alpha i}\right) \frac{r_{\alpha i}^{2}-r_{\beta i}^{2}}{r_{\alpha i}},
\end{gathered}
$$




$$
\begin{aligned}
Z_{3,3}=\frac{1}{2} \sum_{i} \sum_{j \neq i} \sum_{\substack{k \neq i \\
k \neq j}} U^{\prime}\left(n_{i}\right) f\left(r_{i j}\right) f\left(r_{i k}\right) g^{\prime}\left(\cos \theta_{j i k}\right) \delta_{\alpha j} \delta_{\beta i} \frac{\cos \theta_{j i k}}{r_{i j}} \\
=\frac{1}{2} U^{\prime}\left(n_{\beta}\right) \frac{f\left(r_{\beta \alpha}\right)}{r_{\beta \alpha}} \sum_{\substack{i \neq \beta \\
i \neq \alpha}} f\left(r_{\beta i}\right) g^{\prime}\left(\cos \theta_{\alpha \beta i}\right) \cos \theta_{\alpha \beta i},
\end{aligned}
$$

$$
\begin{gathered}
Z_{3,4}=\frac{1}{2} \sum_{i} \sum_{j \neq i} \sum_{\substack{k \neq i \\
k \neq j}} U^{\prime}\left(n_{i}\right) f\left(r_{i j}\right) f\left(r_{i k}\right) g^{\prime}\left(\cos \theta_{j i k}\right) \delta_{\alpha j} \delta_{\beta i}\left(-\frac{r_{i k}^{2}-r_{j k}^{2}}{r_{i k}} \frac{1}{r_{i j}^{2}}\right) \\
=-\frac{1}{2} U^{\prime}\left(n_{\beta}\right) \frac{f\left(r_{\beta \alpha}\right)}{r_{\beta \alpha}^{2}} \sum_{\substack{i \neq \beta \\
i \neq \alpha}} f\left(r_{\beta i}\right) g^{\prime}\left(\cos \theta_{\alpha \beta i}\right) \frac{r_{\beta i}^{2}-r_{\alpha i}^{2}}{r_{\beta i}},
\end{gathered}
$$

$$
\begin{aligned}
Z_{3,5}=\frac{1}{2} \sum_{i} \sum_{j \neq i} \sum_{\substack{k \neq i \\
k \neq j}} U^{\prime}\left(n_{i}\right) f\left(r_{i j}\right) f\left(r_{i k}\right) g^{\prime}\left(\cos \theta_{j i k}\right) \delta_{\alpha i} \delta_{\beta k} \frac{\cos \theta_{j i k}}{r_{i k}} \\
=\frac{1}{2} U^{\prime}\left(n_{\alpha}\right) \frac{f\left(r_{\alpha \beta}\right)}{r_{\alpha \beta}} \sum_{\substack{i \neq \alpha \\
i \neq \beta}} f\left(r_{\alpha i}\right) g^{\prime}\left(\cos \theta_{i \alpha \beta}\right) \cos \theta_{i \alpha \beta}
\end{aligned}
$$

$$
\begin{gathered}
Z_{3,6}=\frac{1}{2} \sum_{i} \sum_{j \neq i} \sum_{\substack{k \neq i \\
k \neq j}} U^{\prime}\left(n_{i}\right) f\left(r_{i j}\right) f\left(r_{i k}\right) g^{\prime}\left(\cos \theta_{j i k}\right) \delta_{\alpha i} \delta_{\beta k}\left(-\frac{r_{i j}^{2}-r_{j k}^{2}}{r_{i j}} \frac{1}{r_{i k}^{2}}\right) \\
=-\frac{1}{2} U^{\prime}\left(n_{\alpha}\right) \frac{f\left(r_{\alpha \beta}\right)}{r_{\alpha \beta}^{2}} \sum_{\substack{i \neq \alpha \\
i \neq \beta}} f\left(r_{\alpha i}\right) g^{\prime}\left(\cos \theta_{i \alpha \beta}\right) \frac{r_{\alpha i}^{2}-r_{i \beta}^{2}}{r_{\alpha i}},
\end{gathered}
$$

$$
\begin{array}{r}
Z_{3,7}=\frac{1}{2} \sum_{i} \sum_{j \neq i} \sum_{\substack{k \neq i \\
k \neq j}} U^{\prime}\left(n_{i}\right) f\left(r_{i j}\right) f\left(r_{i k}\right) g^{\prime}\left(\cos \theta_{j i k}\right) \delta_{\alpha k} \delta_{\beta i} \frac{\cos \theta_{j i k}}{r_{i k}} \\
=\frac{1}{2} U^{\prime}\left(n_{\beta}\right) \frac{f\left(r_{\beta \alpha}\right)}{r_{\beta \alpha}} \sum_{\substack{i \neq \beta \\
i \neq \alpha}} f\left(r_{\beta i}\right) g^{\prime}\left(\cos \theta_{i \beta \alpha}\right) \cos \theta_{i \beta \alpha}
\end{array}
$$

$$
\begin{gathered}
Z_{3,8}=\frac{1}{2} \sum_{i} \sum_{j \neq i} \sum_{\substack{k \neq i \\
k \neq j}} U^{\prime}\left(n_{i}\right) f\left(r_{i j}\right) f\left(r_{i k}\right) g^{\prime}\left(\cos \theta_{j i k}\right) \delta_{\alpha k} \delta_{\beta i}\left(-\frac{r_{i j}^{2}-r_{j k}^{2}}{r_{i j}} \frac{1}{r_{i k}^{2}}\right) \\
=-\frac{1}{2} U^{\prime}\left(n_{\beta}\right) \frac{f\left(r_{\beta \alpha}\right)}{r_{\beta \alpha}^{2}} \sum_{\substack{i \neq \beta \\
i \neq \alpha}} f\left(r_{\beta i}\right) g^{\prime}\left(\cos \theta_{i \beta \alpha}\right) \frac{r_{\beta i}^{2}-r_{i \alpha}^{2}}{r_{\beta i}},
\end{gathered}
$$

$$
\begin{gathered}
Z_{3,9}=\frac{1}{2} \sum_{i} \sum_{j \neq i} \sum_{\substack{k \neq i \\
k \neq j}} U^{\prime}\left(n_{i}\right) f\left(r_{i j}\right) f\left(r_{i k}\right) g^{\prime}\left(\cos \theta_{j i k}\right) \delta_{\alpha j} \delta_{\beta k}\left(-\frac{r_{j k}}{r_{i j} r_{i k}}\right) \\
=-\frac{1}{2} r_{\alpha \beta} \sum_{\substack{i \neq \alpha \\
i \neq \beta}} U^{\prime}\left(n_{i}\right) \frac{f\left(r_{i \alpha}\right)}{r_{i \alpha}} \frac{f\left(r_{i \beta}\right)}{r_{i \beta}} g^{\prime}\left(\cos \theta_{\alpha i \beta}\right)
\end{gathered}
$$


and

$$
\begin{gathered}
Z_{3,10}=\frac{1}{2} \sum_{i} \sum_{\substack{j \neq i \\
\text { a }}} \sum_{\substack{k \neq i \\
k \neq j}} U^{\prime}\left(n_{i}\right) f\left(r_{i j}\right) f\left(r_{i k}\right) g^{\prime}\left(\cos \theta_{j i k}\right) \delta_{\alpha k} \delta_{\beta j}\left(-\frac{r_{j k}}{r_{i j} r_{i k}}\right) \\
=-\frac{1}{2} r_{\beta \alpha} \sum_{\substack{i \neq \beta \\
i \neq \alpha}} U^{\prime}\left(n_{i}\right) \frac{f\left(r_{i \beta}\right)}{r_{i \beta}} \frac{f\left(r_{i \alpha}\right)}{r_{i \alpha}} g^{\prime}\left(\cos \theta_{\beta i \alpha}\right) .
\end{gathered}
$$

In each of the formulae above we employed the properties of the Kronecker delta and renamed dummy indices so that only $\alpha, \beta$, and $i$ remain.

By a pairwise combining of the expressions (32)-(41), we can significantly simplify the long-winded expression for $Z_{3}$. To wit, by adding (32) and (36) we obtain

$$
Z_{3,1}+Z_{3,5}=U^{\prime}\left(n_{\alpha}\right) \frac{f\left(r_{\alpha \beta}\right)}{r_{\alpha \beta}} \sum_{\substack{i \neq \alpha \\ i \neq \beta}} f\left(r_{\alpha i}\right) g^{\prime}\left(\cos \theta_{\beta \alpha i}\right) \cos \theta_{\beta \alpha i} .
$$

By adding (34) and (38) we obtain

$$
Z_{3,3}+Z_{3,7}=U^{\prime}\left(n_{\beta}\right) \frac{f\left(r_{\beta \alpha}\right)}{r_{\beta \alpha}} \sum_{\substack{i \neq \beta \\ i \neq \alpha}} f\left(r_{\beta i}\right) g^{\prime}\left(\cos \theta_{\alpha \beta i}\right) \cos \theta_{\alpha \beta i} .
$$

By adding (33) and (37) we obtain

$$
Z_{3,2}+Z_{3,6}=-U^{\prime}\left(n_{\alpha}\right) \frac{f\left(r_{\alpha \beta}\right)}{r_{\alpha \beta}^{2}} \sum_{\substack{i \neq \alpha \\ i \neq \beta}} \frac{f\left(r_{\alpha i}\right)}{r_{\alpha i}} g^{\prime}\left(\cos \theta_{\beta \alpha i}\right)\left(r_{\alpha i}^{2}-r_{\beta i}^{2}\right) .
$$

By adding (35) and (39) we obtain

$$
Z_{3,4}+Z_{3,8}=-U^{\prime}\left(n_{\beta}\right) \frac{f\left(r_{\beta \alpha}\right)}{r_{\beta \alpha}^{2}} \sum_{\substack{i \neq \beta \\ i \neq \alpha}} \frac{f\left(r_{\beta i}\right)}{r_{\beta i}} g^{\prime}\left(\cos \theta_{\alpha \beta i}\right)\left(r_{\beta i}^{2}-r_{\alpha i}^{2}\right),
$$

and by adding (40) and (41) we obtain

$$
Z_{3,9}+Z_{3,10}=-r_{\alpha \beta} \sum_{\substack{i \neq \alpha \\ i \neq \beta}} U^{\prime}\left(n_{i}\right) \frac{f\left(r_{i \alpha}\right)}{r_{i \alpha}} \frac{f\left(r_{i \beta}\right)}{r_{i \beta}} g^{\prime}\left(\cos \theta_{\alpha i \beta}\right) .
$$

In the above we used $r_{i j}=r_{j i}$ and $\cos \theta_{j i k}=\cos \theta_{k i j}$.

By invoking the cosine rule again in the form of

$$
r_{\alpha i}^{2}-r_{\beta i}^{2}=2 r_{\alpha \beta} r_{\alpha i} \cos \theta_{\beta \alpha i}-r_{\alpha \beta}^{2},
$$

we can further simplify $Z_{3}$ by combining (42) with (44)

$$
\begin{aligned}
\left(Z_{3,1}+Z_{3,5}\right)+ & \left(Z_{3,2}+Z_{3,6}\right)=U^{\prime}\left(n_{\alpha}\right) \frac{f\left(r_{\alpha \beta}\right)}{r_{\alpha \beta}} \sum_{\substack{i \neq \alpha \\
i \neq \beta}} f\left(r_{\alpha i}\right) g^{\prime}\left(\cos \theta_{\beta \alpha i}\right) \cos \theta_{\beta \alpha i} \\
& -U^{\prime}\left(n_{\alpha}\right) \frac{f\left(r_{\alpha \beta}\right)}{r_{\alpha \beta}^{2}} \sum_{\substack{i \neq \alpha \\
i \neq \beta}} \frac{f\left(r_{\alpha i}\right)}{r_{\alpha i}} g^{\prime}\left(\cos \theta_{\beta \alpha i}\right)\left(r_{\alpha i}^{2}-r_{\beta i}^{2}\right) \\
& =U^{\prime}\left(n_{\alpha}\right) \frac{f\left(r_{\alpha \beta}\right)}{r_{\alpha \beta}} \sum_{\substack{i \neq \alpha \\
i \neq \beta}} f\left(r_{\alpha i}\right) g^{\prime}\left(\cos \theta_{\beta \alpha i}\right) \cos \theta_{\beta \alpha i} \\
& -U^{\prime}\left(n_{\alpha}\right) \frac{f\left(r_{\alpha \beta}\right)}{r_{\alpha \beta}^{2}} \sum_{\substack{i \neq \alpha \\
i \neq \beta}} \frac{f\left(r_{\alpha i}\right)}{r_{\alpha i}} g^{\prime}\left(\cos \theta_{\beta \alpha i}\right)\left(2 r_{\alpha \beta} r_{\alpha i} \cos \theta_{\beta \alpha i}-r_{\alpha \beta}^{2}\right)
\end{aligned}
$$




$$
\begin{aligned}
& =U^{\prime}\left(n_{\alpha}\right) f\left(r_{\alpha \beta}\right) \sum_{\substack{i \neq \alpha \\
i \neq \beta}} \frac{f\left(r_{\alpha i}\right)}{r_{\alpha i}} g^{\prime}\left(\cos \theta_{\beta \alpha i}\right) \\
& -U^{\prime}\left(n_{\alpha}\right) \frac{f\left(r_{\alpha \beta}\right)}{r_{\alpha \beta}} \sum_{\substack{i \neq \alpha \\
i \neq \beta}} f\left(r_{\alpha i}\right) g^{\prime}\left(\cos \theta_{\beta \alpha i}\right) \cos \theta_{\beta \alpha i},
\end{aligned}
$$

and (43) with (45)

$$
\begin{gathered}
\left(Z_{3,3}+Z_{3,7}\right)+\left(Z_{3,4}+Z_{3,8}\right)=U^{\prime}\left(n_{\beta}\right) f\left(r_{\beta \alpha}\right) \sum_{\substack{i \neq \beta \\
i \neq \alpha}} \frac{f\left(r_{\beta i}\right)}{r_{\beta i}} g^{\prime}\left(\cos \theta_{\alpha \beta i}\right) \\
-U^{\prime}\left(n_{\beta}\right) \frac{f\left(r_{\beta \alpha}\right)}{r_{\beta \alpha}} \sum_{\substack{i \neq \beta \\
i \neq \alpha}} f\left(r_{\beta i}\right) g^{\prime}\left(\cos \theta_{\alpha \beta i}\right) \cos \theta_{\alpha \beta i} .
\end{gathered}
$$

This completes the simplification of $Z_{3}$, which, owing to (46), (48), and (49) can be written in its final form:

$$
\begin{aligned}
Z_{3}= & U^{\prime}\left(n_{\alpha}\right) f\left(r_{\alpha \beta}\right) \sum_{\substack{i \neq \alpha \\
i \neq \beta}} \frac{f\left(r_{\alpha i}\right)}{r_{\alpha i}} g^{\prime}\left(\cos \theta_{\beta \alpha i}\right) \\
& -U^{\prime}\left(n_{\alpha}\right) \frac{f\left(r_{\alpha \beta}\right)}{r_{\alpha \beta}} \sum_{\substack{i \neq \alpha \\
i \neq \beta}} f\left(r_{\alpha i}\right) g^{\prime}\left(\cos \theta_{\beta \alpha i}\right) \cos \theta_{\beta \alpha i} \\
& +U^{\prime}\left(n_{\beta}\right) f\left(r_{\beta \alpha}\right) \sum_{\substack{i \neq \beta \\
i \neq \alpha}} \frac{f\left(r_{\beta i}\right)}{r_{\beta i}} g^{\prime}\left(\cos \theta_{\alpha \beta i}\right) \\
& -U^{\prime}\left(n_{\beta}\right) \frac{f\left(r_{\beta \alpha}\right)}{r_{\beta \alpha}} \sum_{\substack{i \neq \beta \\
i \neq \alpha}} f\left(r_{\beta i}\right) g^{\prime}\left(\cos \theta_{\alpha \beta i}\right) \cos \theta_{\alpha \beta i} \\
& -r_{\alpha \beta} \sum_{\substack{i \neq \alpha \\
i \neq \beta}} U^{\prime}\left(n_{i}\right) \frac{f\left(r_{i \alpha}\right)}{r_{i \alpha}} \frac{f\left(r_{i \beta}\right)}{r_{i \beta}} g^{\prime}\left(\cos \theta_{\alpha i \beta}\right) .
\end{aligned}
$$

Returning to the original expression (17), and using intermediate results of (20), (21), (23), (24), and (50), we finally obtain

$$
\begin{aligned}
\frac{\partial E_{\mathrm{pot}}}{\partial r_{\alpha \beta}}= & \phi^{\prime}\left(r_{\alpha \beta}\right)+U^{\prime}\left(n_{\alpha}\right) \rho^{\prime}\left(r_{\alpha \beta}\right)+U^{\prime}\left(n_{\beta}\right) \rho^{\prime}\left(r_{\beta \alpha}\right) \\
& +U^{\prime}\left(n_{\alpha}\right) f^{\prime}\left(r_{\alpha \beta}\right) \sum_{\substack{i \neq \alpha \\
i \neq \beta}} f\left(r_{\alpha i}\right) g\left(\cos \theta_{\beta \alpha i}\right) \\
& +U^{\prime}\left(n_{\alpha}\right) f\left(r_{\alpha \beta}\right) \sum_{\substack{i \neq \alpha \\
i \neq \beta}} \frac{f\left(r_{\alpha i}\right)}{r_{\alpha i}} g^{\prime}\left(\cos \theta_{\beta \alpha i}\right) \\
& -U^{\prime}\left(n_{\alpha}\right) \frac{f\left(r_{\alpha \beta}\right)}{r_{\alpha \beta}} \sum_{\substack{i \neq \alpha \\
i \neq \beta}} f\left(r_{\alpha i}\right) g^{\prime}\left(\cos \theta_{\beta \alpha i}\right) \cos \theta_{\beta \alpha i} \\
& +U^{\prime}\left(n_{\beta}\right) f^{\prime}\left(r_{\beta \alpha}\right) \sum_{\substack{i \neq \beta \\
i \neq \alpha}} f\left(r_{\beta i}\right) g\left(\cos \theta_{\alpha \beta i}\right) \\
& +U^{\prime}\left(n_{\beta}\right) f\left(r_{\beta \alpha}\right) \sum_{\substack{i \neq \beta \\
i \neq \alpha}} \frac{f\left(r_{\beta i}\right)}{r_{\beta i}} g^{\prime}\left(\cos \theta_{\alpha \beta i}\right)
\end{aligned}
$$




$$
\begin{aligned}
& -U^{\prime}\left(n_{\beta}\right) \frac{f\left(r_{\beta \alpha}\right)}{r_{\beta \alpha}} \sum_{\substack{i \neq \beta \\
i \neq \alpha}} f\left(r_{\beta i}\right) g^{\prime}\left(\cos \theta_{\alpha \beta i}\right) \cos \theta_{\alpha \beta i} \\
& -r_{\alpha \beta} \sum_{\substack{i \neq \beta \\
i \neq \alpha}} U^{\prime}\left(n_{i}\right) \frac{f^{\prime}\left(r_{i \alpha}\right)}{r_{i \alpha}} \frac{f^{\prime}\left(r_{i \beta}\right)}{r_{i \beta}} g^{\prime}\left(\cos \theta_{\alpha i \beta}\right) .
\end{aligned}
$$

Returning to the original indices $i, j$, and $k$, and employing (14), we can express the force with which particle $j$ acts on particle $i$ as

$$
\mathbf{F}_{i j}=\frac{\mathbf{r}_{i j}}{r_{i j}} \times\left(\phi^{\prime}\left(r_{i j}\right)+\left[U^{\prime}\left(n_{i}\right)+U^{\prime}\left(n_{j}\right)\right] \rho^{\prime}\left(r_{i j}\right)+\Theta_{i j}\right),
$$

where $\Theta_{i j}$ denotes the contributions to the force that have an explicit angular nature. The term $\Theta_{i j}$ is given by

$$
\begin{aligned}
\Theta_{i j}= & U^{\prime}\left(n_{i}\right)\left\{f^{\prime}\left(r_{i j}\right) \sum_{\substack{k \neq i \\
k \neq j}} f\left(r_{i k}\right) g\left(\cos \theta_{j i k}\right)\right. \\
& +f\left(r_{i j}\right) \sum_{\substack{k \neq i \\
k \neq j}} \frac{f\left(r_{i k}\right)}{r_{i k}} g^{\prime}\left(\cos \theta_{j i k}\right) \\
& \left.-\frac{f\left(r_{i j}\right)}{r_{i j}} \sum_{\substack{k \neq i \\
k \neq j}} f\left(r_{i k}\right) g^{\prime}\left(\cos \theta_{j i k}\right) \cos \theta_{j i k}\right\} \\
& +U^{\prime}\left(n_{j}\right)\left\{f^{\prime}\left(r_{j i}\right) \sum_{\substack{k \neq i \\
k \neq j}} f\left(r_{j k}\right) g\left(\cos \theta_{i j k}\right)\right. \\
& +f\left(r_{j i}\right) \sum_{\substack{k \neq i \\
k \neq j}} \frac{f\left(r_{j k}\right)}{r_{j k}} g^{\prime}\left(\cos \theta_{i j k}\right) \\
& \left.-\frac{f\left(r_{j i}\right)}{r_{j i}} \sum_{\substack{k \neq i \\
k \neq j}} f\left(r_{j k}\right) g^{\prime}\left(\cos \theta_{i j k}\right) \cos \theta_{i j k}\right\} \\
& -r_{i j} \sum_{\substack{k \neq i \\
k \neq j}} U^{\prime}\left(n_{k}\right) \frac{f\left(r_{k i}\right)}{r_{k i}} \frac{f\left(r_{k j}\right)}{r_{k j}} g^{\prime}\left(\cos \theta_{i k j}\right) .
\end{aligned}
$$

The expressions (52) and (53) define a central-force decomposition of the s-MEAM potential and constitute the main result of this work.

\section{Discussion}

Below we discuss the properties of the obtained expressions (52)-(53), with particular focus on the term $\Theta_{i j}$. This term is composed of three sub-terms, involving, respectively, $U^{\prime}\left(n_{i}\right), U^{\prime}\left(n_{j}\right)$, and $r_{i j}$ (cf. (53)). For the sake of brevity, we shall denote the sums appearing in (53) as follows:

(i) $\Theta_{j i k}^{(1)}, \Theta_{j i k}^{(2)}, \Theta_{j i k}^{(3)}-$ sums in the $U^{\prime}\left(n_{i}\right)$ term,

(ii) $\Theta_{i j k}^{(1)}, \Theta_{i j k}^{(2)}, \Theta_{i j k}^{(3)}-$ sums in the $U^{\prime}\left(n_{j}\right)$ term, 
(iii) $\Theta_{i k j}^{(4)}$ - the sum in the $r_{i j}$ term.

Using this shortcut notation we can rewrite (53) as

$$
\begin{aligned}
\Theta_{i j}= & U^{\prime}\left(n_{i}\right)\left\{f^{\prime}\left(r_{i j}\right) \Theta_{j i k}^{(1)}+f\left(r_{i j}\right) \Theta_{j i k}^{(2)}-\frac{f\left(r_{i j}\right)}{r_{i j}} \Theta_{j i k}^{(3)}\right\} \\
& +U^{\prime}\left(n_{j}\right)\left\{f^{\prime}\left(r_{j i}\right) \Theta_{i j k}^{(1)}+f\left(r_{j i}\right) \Theta_{i j k}^{(2)}-\frac{f\left(r_{j i}\right)}{r_{j i}} \Theta_{i j k}^{(3)}\right\} \\
& -r_{i j} \Theta_{i k j}^{(4)} .
\end{aligned}
$$

The first term, involving $U^{\prime}\left(n_{i}\right)$, describes the central interactions of a particle $i$ with its first-nearest neighbours, which is made apparent by the presence of $f^{\prime}\left(r_{i j}\right)$ and $f\left(r_{i j}\right)$ (which are non-zero only for $j \in F(i)$ ) in front of each of the sums $\Theta_{j i k}^{(1)}$, $\Theta_{j i k}^{(2)}$, and $\Theta_{j i k}^{(3)}$. We note that the contribution to the central force on $i$ from a firstnearest neighbour $j \in F(i)$ depends on the positions of all first-nearest neighbours of $i$, because $f\left(r_{i k}\right)$ (which is non-zero for all $k \in F(i)$ ) is present in $\Theta_{j i k}^{(1)}, \Theta_{j i k}^{(2)}$, and $\Theta_{j i k}^{(3)}$.

The second term, involving $U^{\prime}\left(n_{j}\right)$, also describes the central interactions of a particle $i$ with its first-nearest neighbours $j \in F(i)$, which is made apparent by the presence of $f^{\prime}\left(r_{j i}\right)$ and $f\left(r_{j i}\right)$ in front of each of the sums $\Theta_{i j k}^{(1)}, \Theta_{i j k}^{(2)}$, and $\Theta_{i j k}^{(3)}$. The crucial difference in this case is that the contribution depends on the positions of the first-nearest neighbours of $j$, rather than those of $i$, due to the presence of $f\left(r_{j k}\right)$, which is non-zero for all $k \in F(j)$, in the sums $\Theta_{i j k}^{(1)}, \Theta_{i j k}^{(2)}$, and $\Theta_{i j k}^{(3)}$.

The third term, involving $r_{i j}$, captures the contributions to $\mathbf{F}_{i j}$ from particles that are first-nearest neighbours of both $i$ and $j$. This is made apparent by the presence of $f\left(r_{k i}\right)$ and $f\left(r_{k j}\right)$ in the sum $\Theta_{i k j}^{(4)}$. We note that the particles that are first-nearest neighbours of both $i$ and $j$ continue contributing to $\mathbf{F}_{i j}$ through the first and second terms of (53).

It is also instructive to examine expression (53) with the aim of identifying, for a chosen atom $i$, all atoms $j$ that interact centrally with $i$, i.e. those for which $\mathbf{F}_{i j}$ is non-vanishing. Here we do not make any assumptions a priori on the membership of $j$ in $F(i), S(i)$ or $B(i)$. We focus our attention on the last term in (53), and in particular on the product $f\left(r_{k i}\right) f\left(r_{k j}\right)$ appearing under the sum. This product is, of course, non-zero for those atoms $j$, for which there exists an atom $k$ for which both $f\left(r_{k i}\right)$ and $f\left(r_{k j}\right)$ are non-zero. This means that apart from interacting centrally with atoms $j$ that are its first-nearest neighbours (which is a consequence of the first and second terms of (53), as explained earlier), atom $i$ also interacts centrally with atoms $j$ that are its second-nearest neighbours, i.e. with $j \in S(i)$. We can also express this observation as follows: if any atom $j$ is a first-nearest neighbour of an atom $k$ $\left(f\left(r_{k j}\right) \neq 0\right)$ such that $k$ is also a first-nearest neighbour of $i\left(f\left(r_{k i}\right) \neq 0\right)$, then the central force $\mathbf{F}_{i j}$ is non-vanishing. This is another way of restating the fact that atom $i$ interacts centrally also with first-neighbours of its first-neighbours. We note in passing that for atoms that are second-nearest neighbours of $i$, i.e. $j \in S(i)$, which implies $f\left(r_{i j}\right)=0$, the expression (53) simplifies to:

$$
\Theta_{i j}=-r_{i j} \sum_{\substack{k \neq i \\ k \neq j}} U^{\prime}\left(n_{k}\right) \frac{f\left(r_{k i}\right)}{r_{k i}} \frac{f\left(r_{k j}\right)}{r_{k j}} g^{\prime}\left(\cos \theta_{i k j}\right) .
$$

We point out an important consequence of the above observations. Despite the fact that the definitions of the functions $\phi(r), \rho(r)$, and $f(r)$ explicitly include a cutoff 
radius $r_{\mathrm{c}}$, an s-MEAM-class potential has an effective cutoff radius (in the sense of a non-vanishing central forces) of $2 r_{\mathrm{c}}$.

\section{Example application}

We will now demonstrate the applicability of the CFD expressions for the s-MEAM potential derived in this work on an example problem of determining the stress field generated by a dislocation. Our choice of problem is motivated by its relevance in the investigations of mechanical properties of the solid state, and by the fact that, over decades, it has proved challenging for theoretical approaches [69,70], particularly when the stress field around the dislocation core is of interest. For a review of the issues the interested Reader is referred to [71].

For this and similar problems particle methods constitute a viable alternative to analytical approaches, as they explicitly take the granularity of matter into account, along with the specifics of interatomic interactions [72]. To obtain a faithful description of the phenomena taking place in the immediate vicinity of a dislocation it is necessary to employ a highly transferable potential. However, Hardy's formalism can only be used in conjunction with relatively simple potentials for which CFD's are available today. Below we demonstrate how the decomposition derived in this work can be used to obtain a realistic description of stress fields, owing to the improved accuracy that s-MEAM potentials offer for structures that are far from equilibrium [11,22,31,32].

\subsection{Computational set-up}

Our test case was an edge dislocation with a Burgers vector $\mathbf{b}=1 / 2\langle 111\rangle$ in a bcc monocrystal of molybdenum. We employ molecular statics [73] (MS) in conjunction with the periodic array of dislocations (PAD) method [74]. This approach, proposed by Baskes and coworkers [25,75], has found widespread use in the investigation of line defects [76-86], both in the study of statical properties of dislocations (structure and energetics of the dislocation core, stress and strain fields due to dislocations), and of their dynamical properties (dislocation motion and ensuing slip).

PAD serves as a protocol for constructing a system containing a dislocation together with a suitable definition of boundary conditions. The relevant construct is shown in figure 1 and will be briefly discussed below. A more detailed description of PAD, along with a discussion of its applicability and limitations can be found in [74].

We began by generating a cuboid block of bcc Mo, adopting a reference frame where the $x$ axis is parallel to [111] and the $y$ axis is parallel to [11 $]$, which corresponds to the $z$ axis being aligned with $[\overline{1} 10]$. We assumed $a=3.167 \AA$ for the lattice constant, based on the equilibrium value for $T=0 \mathrm{~K}$ in the s-MEAM description [22]. The dimensions of the block were taken to be $L_{x}=100 \sqrt{3} a, L_{y}=8 \sqrt{6} a$, and $L_{z}=124 \sqrt{2} a$. An edge dislocation was subsequently introduced by cleaving the monocrystal along the $z=L_{z} / 2$ plane, the removal of three crystallographic halfplanes (111) from the bottom half-crystal, and matching the resultant half-crystals by uniform deformation from $L_{x}$ for the top half-crystal and from $L_{x}-b$ for the bottom half-crystal to achieve a final dimension of $L_{x}-b / 2$ for both. The resultant system measured $L_{x}=547.241 \AA$ by $L_{y}=62.068 \AA$ by $L_{z}=555.445 \AA$ and comprised $N=1187424$ atoms.

The above procedure generates an edge dislocation with a Burgers vector of $\mathbf{b}=[\sqrt{3} / 2 a, 0,0]$ and a dislocation line parallel to the $y$ axis. At this stage the 
(a)

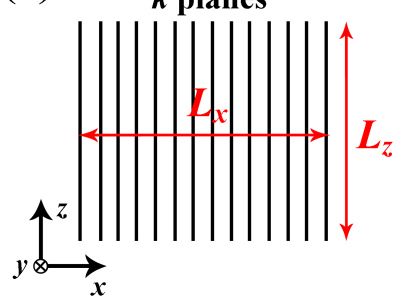

(c)

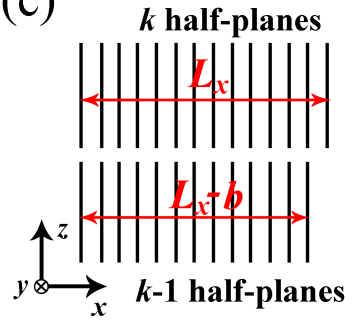

(b)

$\boldsymbol{k}$ half-planes

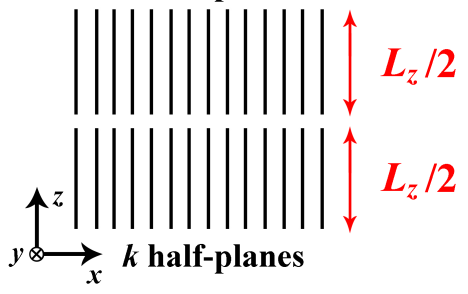

(d)

$\boldsymbol{k}$ half-planes
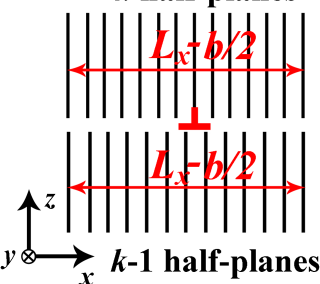

(e)

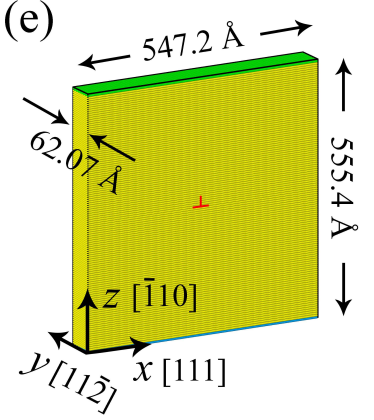

Figure 1. System set-up in the PAD method. Panels (a)-(d) illustrate the procedure for generating a system with a dislocation. Solid lines represent crystallographic planes, while $\perp$ denotes the position of the introduced dislocation. The monocrystal is (a) cleaved to create two half-crystals (b). A number of crystallographic planes is (c) removed from the bottom half-crystal. The final step (d) involves matching the linear dimensions of both half-crystals. Panel (e) presents the final set-up.

dislocation is not physically realistic, which becomes apparent when the strain fields are examined. In order to reach a more plausible state, in the last stage of the protocol we performed energy minimisation on the system. Consistent with the PAD approach, periodic boundary conditions (PBCs) were employed along $x$ and $y$ axes only, corresponding to simulating a periodic array of quasi-infinitely long dislocations separated by $L_{x}$, located in a finite-width slab of material. As the dimensions of the system are increased $\left(L_{x}, L_{z} \rightarrow \infty\right)$, the interaction of the dislocation with its periodic images and the influence of the two free surfaces $z=0$ and $z=L_{z}$ becomes negligible, with the behaviour of the system converging to that of a single, infinitely long dislocation in an otherwise ideal monocrystal. It has been shown [74] that such systems are sufficiently converged already at $L_{x}, L_{z} \approx 200 b$, which in our case (a dislocation with $\mathbf{b}=1 / 2\langle 111\rangle$ in molybdenum) corresponds to $L_{x}, L_{z} \approx 548 \AA$, 
guiding our choice of system dimensions.

Energy minimisation was performed with atoms lying in the outermost crystallographic planes $(\overline{1} 10)$ (i.e. those with $z=0$ or $z=L_{z}$ ) constrained to move only in the $x y$ plane. The termination criterion was for all atomic force components to be below $3 \times 10^{-5} \mathrm{eV} / \AA$, with final positions being accurate to no worse than $10^{-6} \AA$. We used the s-MEAM potential of Park et al [22], for which the cutoff radius is $r_{\mathrm{c}}=5.9 \AA$. Minimisation was carried out using LAMMPS [68] with the Polak-Ribière [87] formulation of the conjugate gradient method.

Production calculations consisted in calculating the dislocation's Hardy stress field by post-processing the configuration obtained earlier from energy minimisation. First, all non-zero central forces $\mathbf{F}_{i j}$ were determined from (52) and (53). Subsequently, Hardy's formalism (equations (7)-(10)) was leveraged to calculate the spatial distribution of the stress tensor $\boldsymbol{\sigma}(\mathbf{x})$. Since all calculations shown here correspond to $T=0 \mathrm{~K}$, we set $\boldsymbol{\sigma}^{\mathrm{K}}(\mathbf{x}) \equiv 0$ in $(7)$. The kernel functions were taken to be Gaussians truncated at $r_{\mathrm{w}}$, i.e.

$$
w(\mathbf{x}, \mathbf{y})=\left\{\begin{array}{ll}
\frac{1}{(\sqrt{\pi} h)^{3}} \exp \left(-\frac{|\mathbf{x}-\mathbf{y}|^{2}}{h^{2}}\right), & |\mathbf{x}-\mathbf{y}| \leq r_{\mathrm{w}} \\
0, & |\mathbf{x}-\mathbf{y}|>r_{\mathrm{w}}
\end{array} .\right.
$$

We chose the truncation radius $r_{\mathrm{w}}$ as twice the cutoff radius, i.e. $r_{\mathrm{w}}=11.8 \AA$. For this choice the kernel function at truncation radius is $w(\mathbf{x}, \mathbf{y})=3.3 \times 10^{-6}$, which means that the truncation has negligible effect on the calculated stress distribution.

It is recognized that the details of the stress distribution obtained from Hardy's formalism depend, to a degree, on the choice of the kernel function smoothing length $h$. We verified through numerical tests that for the value of $r_{\mathrm{w}}$ given above, satisfactory results are obtained for $h \in\left[0.2 r_{\mathrm{w}}, 0.5 r_{\mathrm{w}}\right]$. Smaller values lead to excessive sharpening, yielding a grainy distribution, where the discrete nature of the material becomes apparent. Larger values, on the other hand, result in excessive smoothing, discarding a non-negligible fraction of $\boldsymbol{\sigma}$, and making the distribution overly blurred. Following Shen and Atluri [50] we set the smoothing length to $h=0.4 r_{\mathrm{w}}=4.72 \AA$, and all results we report in plots use that value. The above choice yields an image of stress distribution that is simultaneously sufficiently fine-grained and smooth. While the qualitative picture of the stress distribution does not change for values of $h$ in this ,,reasonable" interval $\left[0.2 r_{\mathrm{w}}, 0.5 r_{\mathrm{w}}\right]$, the particular values at the minima and maxima, and similarly their positions, are more sensitive to this choice. To highlight this, and to give an idea of the magnitude of this sensitivity, we augment the corresponding numerical values (cf. table 1 ) obtained with $h=0.4 r_{\mathrm{w}}$ with values obtained by choosing $h=0.2 r_{\mathrm{w}}$ and $h=0.5 r_{\mathrm{w}}$.

Below we focus our attention on stresses in the dislocation core itself and in its vicinity, calculating $\boldsymbol{\sigma}(\mathbf{x})$ for points $\mathbf{x}=[x, y, z]$ lying in a cuboid region centered on the dislocation line, i.e.:

$$
\begin{aligned}
& L_{x} / 2-15 b \leq x \leq L_{x} / 2+15 b \\
& 0 \leq y<\sqrt{6} a \\
& L_{z} / 2-15 b \leq z \leq L_{z} / 2+15 b .
\end{aligned}
$$

We calculated the stresses on a Cartesian grid, dividing the domain in the $x z$ plane into $121 \times 121$ equispaced points, corresponding to a grid spacing of $b / 4=0.686 \AA$. We investigated the stress distributions $\boldsymbol{\sigma}(\mathbf{x})$ for 60 planes $y=$ const, with the values of $y$ equispaced on the characteristic length $\sqrt{6} a$, leading to a plane spacing of $0.129 \AA$. 
All the distributions presented below correspond to values of $\boldsymbol{\sigma}(\mathbf{x})$ averaged over these planes.

Before we present the obtained stresses, we briefly comment on the overall computational effort of the calculations we performed. Our implementation leveraged symmetric multiprocessing (SMP) through the use of OpenMP [88]. We used a single node with two 12-core Intel Xeon E5 v3 processors @2.3 GHz. The total computational cost was $12.8 \mathrm{~h}$, which corresponds to a (single-core) time of $1.26 \mathrm{~s}$ needed to calculate the stress tensor for each of $121 \times 60 \times 121$ points involved in the calculation.
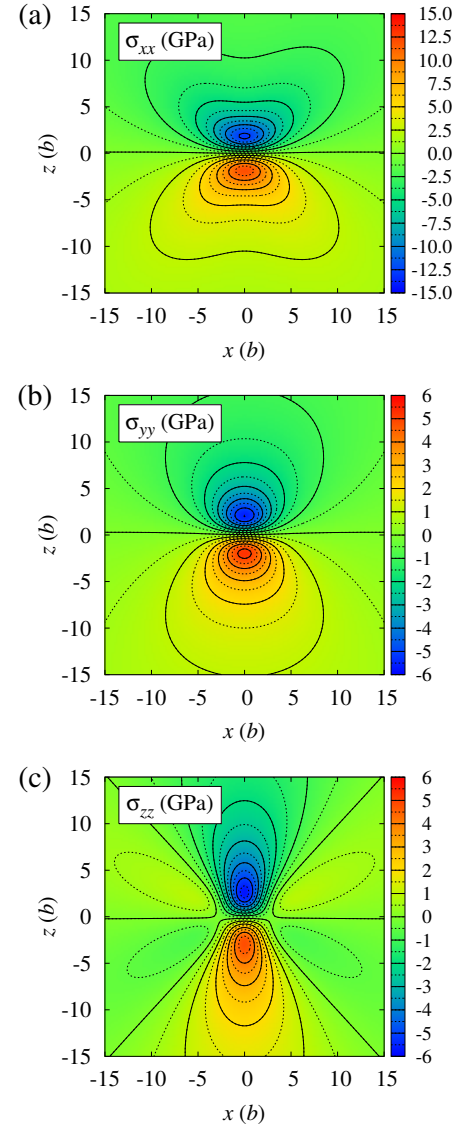
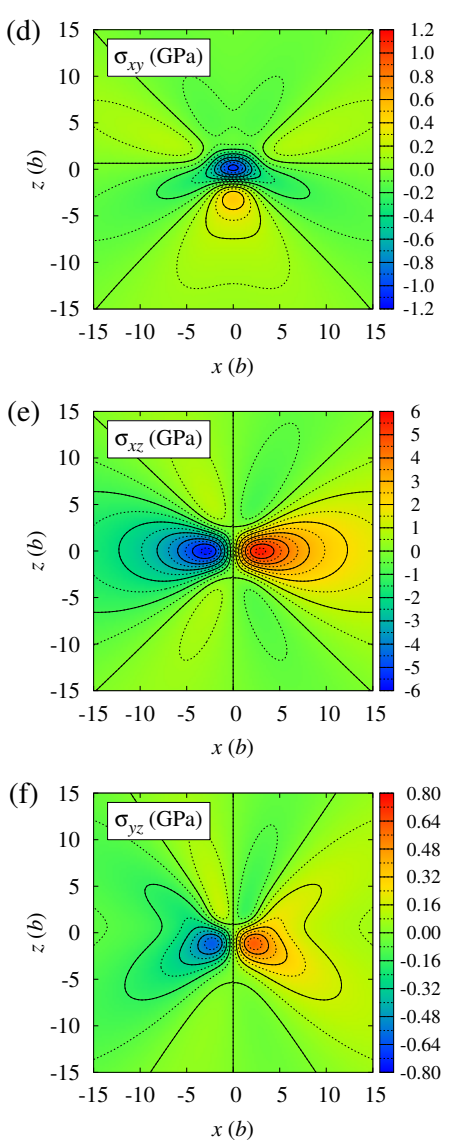

Figure 2. Stress distribution due to an edge dislocation with a Burgers vector $\mathbf{b}=1 / 2\langle 111\rangle$ in a bcc monocrystal of Mo. Each panel corresponds to a different component of the stress tensor. The plane of the figure is the $x z$ plane, with the Burgers vector $\mathbf{b}$ parallel to the $x$ axis, and the dislocation line perpendicular to the plane of the figure, pointing away from the observer. The reference frame was chosen so that the point $(x, z)=(0,0)$ corresponds to the location of the dislocation line. Each panel depicts the distribution of the stress tensor components in a region with dimensions $30 b \times 30 b$ located in the vicinity of the dislocation line. Both axes use the length of the Burgers vector $b=|\mathbf{b}|$ as units. Major (solid) and minor (dashed) isolines were drawn as a guide for the eye. Major isolines correspond to values shown in the legend bar, while minor isolines correspond to median values of two consecutive major isolines. 
Table 1. Positions and values of extrema in $\boldsymbol{\sigma}$. Positions are given as multiples of the Burgers vector length, $b$. Results obtained for various values of the kernel function smoothing length $h$ are presented.

\begin{tabular}{|c|c|c|c|c|c|c|c|}
\hline \multirow[b]{3}{*}{ Component } & \multirow[b]{3}{*}{$h\left(r_{\mathrm{w}}\right)$} & \multicolumn{3}{|c|}{ Minimum } & \multicolumn{3}{|c|}{ Maximum } \\
\hline & & \multirow{2}{*}{$\begin{array}{l}\text { Value } \\
\text { (GPa) }\end{array}$} & \multicolumn{2}{|c|}{ Position } & \multirow{2}{*}{$\begin{array}{l}\text { Value } \\
\text { (GPa) }\end{array}$} & \multicolumn{2}{|c|}{ Position } \\
\hline & & & $x$ & $z$ & & $x$ & $z$ \\
\hline \multirow{3}{*}{$\sigma_{x x}$} & 0.2 & -23.3 & 0 & 1 & 20.5 & 0 & -1 \\
\hline & 0.4 & -12.8 & 0 & 1.75 & 12.0 & 0 & -1.75 \\
\hline & 0.5 & -10.3 & 0 & 2.25 & 9.73 & 0 & -2.25 \\
\hline \multirow{3}{*}{$\sigma_{y y}$} & 0.2 & -9.03 & 0 & 1.25 & 8.25 & 0 & -1 \\
\hline & 0.4 & -5.51 & 0 & 2 & 5.24 & 0 & -2 \\
\hline & 0.5 & -4.52 & 0 & 2.5 & 4.31 & 0 & -2.5 \\
\hline \multirow{3}{*}{$\sigma_{z z}$} & 0.2 & -8.78 & 0 & 1.5 & 7.48 & 0 & -1.75 \\
\hline & 0.4 & -5.71 & 0 & 2.5 & 4.81 & 0 & -2.75 \\
\hline & 0.5 & -4.71 & 0 & 3.25 & 4.02 & 0 & -3.5 \\
\hline \multirow{3}{*}{$\sigma_{x y}$} & 0.2 & -3.46 & 0 & 0 & 1.14 & 0 & -1.75 \\
\hline & 0.4 & -1.05 & 0 & 0.25 & 0.493 & 0 & -3 \\
\hline & 0.5 & -0.691 & 0 & 0.25 & 0.382 & 0 & -3.75 \\
\hline \multirow{3}{*}{$\sigma_{x z}$} & 0.2 & -9.55 & -1.75 & 0 & 9.55 & 1.75 & 0 \\
\hline & 0.4 & -5.64 & -3 & 0 & 5.64 & 3 & 0 \\
\hline & 0.5 & -4.59 & -3.5 & 0 & 4.59 & 3.5 & 0 \\
\hline \multirow{3}{*}{$\sigma_{y z}$} & 0.2 & -1.36 & -1.25 & -0.75 & 1.36 & 1.25 & -0.75 \\
\hline & 0.4 & -0.611 & -2.25 & -1.25 & 0.611 & 2.25 & -1.25 \\
\hline & 0.5 & -0.472 & -2.75 & -1.25 & 0.472 & 2.75 & -1.25 \\
\hline
\end{tabular}

\subsection{Results}

Figure 2 presents two-dimensional maps of the distribution of all six components of the stress tensor $\boldsymbol{\sigma}(\mathbf{x})$. The general character of the obtained distributions is typical and similar to well-known results obtained from analytical solutions of linear elasticity theory (cf. e.g. [69,70] and equations (58)-(62)). The crucial distinguishing feature of the numerical results is the absence of singularities that arise in continuum solutions at $(x, z)=(0,0)$. Here, in contrast, each component of the stress tensor is found to be bounded. We list the positions of the extrema and the corresponding values in table 1 for completeness.

Visual examination of figure 2 reveals the presence of reflection symmetry $x \rightarrow-x$ in the stress distributions, which is well-known in continuum solutions. A more thorough analysis exposes certain asymmetries in the distribution, for example, the subtle breaking of $z \rightarrow-z$ symmetry, which is evidenced by the values and positions of extrema (cf. Tab 1). This effect is modest for $\sigma_{x x}, \sigma_{y y}, \sigma_{z z}$, and $\sigma_{x z}$ due to the large magnitudes of these components. In contrast, for $\sigma_{x y}$ and $\sigma_{y z}$, whose magnitudes are smaller, the effect is much more pronounced - this is easily observed in panels (d) and (f) in figure 2. The reason for the symmetry breaking is the asymmetric structure of the dislocation core relative to the slip plane, illustrated in figure 3 .

We note that the range of variability of $\sigma_{x y}$ and $\sigma_{y z}$ was rather modest - for example on the boundary of the considered region $30 b \times 30 b$ the magnitude of $\sigma_{x y}$ did not exceed $0.116 \mathrm{GPa}$, and the magnitude of $\sigma_{y z}$ did not exceed $0.124 \mathrm{GPa}$. The observation that these components have small magnitudes even relatively close to 
the dislocation line is compatible with the predictions of continuum theory (compare e.g. (62)).

We finish our analysis by comparing the obtained distributions of $\boldsymbol{\sigma}(\mathbf{x})$ with the predictions of linear elasticity theory for an infinitely long edge dislocation in an isotropic medium, where the stress field is given by the following expressions $[69,70]$ :

$$
\begin{aligned}
& \sigma_{x x}(x, z)=-\frac{\mu b}{2 \pi(1-\nu)} z \frac{3 x^{2}+z^{2}}{\left(x^{2}+z^{2}\right)^{2}}, \\
& \sigma_{z z}(x, z)=\frac{\mu b}{2 \pi(1-\nu)} z \frac{x^{2}-z^{2}}{\left(x^{2}+z^{2}\right)^{2}}, \\
& \sigma_{x z}(x, z)=\frac{\mu b}{2 \pi(1-\nu)} x \frac{x^{2}-z^{2}}{\left(x^{2}+z^{2}\right)^{2}}, \\
& \sigma_{y y}(x, z)=\nu\left[\sigma_{x x}(x, z)+\sigma_{z z}(x, z)\right]=-\frac{\mu b \nu}{\pi(1-\nu)} \frac{z}{x^{2}+z^{2}}, \\
& \sigma_{x y}(x, z)=\sigma_{y z}(x, z)=0,
\end{aligned}
$$

where $\mu$ is the shear modulus, and $\nu$ is the Poisson's ratio.

To facilitate the comparison, we plot the components of $\boldsymbol{\sigma}(\mathbf{x})$ for selected crosssections of the system: $x=$ const $=D$ (figure 4 ), and $z=$ const $=D$ (figure 5 ), for $D=0 b, D=7.5 b$, and $D=15 b$. Solid lines correspond to the numerical results obtained from Hardy stress calculations employing the CFD of s-MEAM derived in this work, while dotted lines correspond to analytical (continuum) results of (58)(61). For the latter calculations we used $\mu=120 \mathrm{GPa}$, and $\nu=0.310$, corresponding to experimental data of [90].

Our comparison is perforce strictly qualitative, as the derivation of equations (58)-(61) assumes an isotropic medium. Molybdenum, despite its cubic structure, is characterised by significant anisotropy, as evidenced by a Zener anisotropy

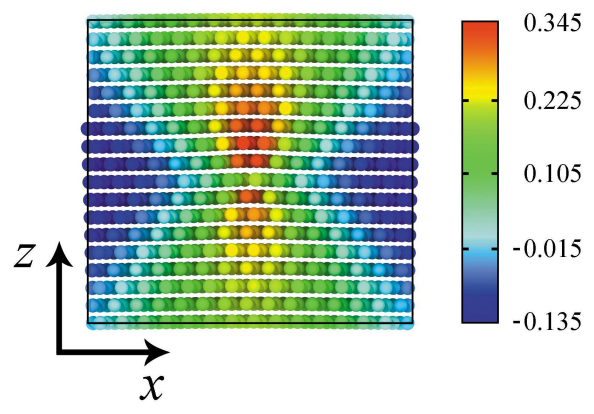

Figure 3. Visualisation of the structure of the dislocation core and its immediate vicinity. The fragment shown corresponds to a region with dimensions $15 b \times 15 b$ centered on the dislocation line. The plane of the figure is the $x z$ plane, with the Burgers vector $\mathbf{b}$ parallel to the $x$ axis, and the dislocation line perpendicular to the plane of the figure, pointing away from the observer. As a guide for the eye atoms were coloured according to their displacement along $z$, with blue corresponding to $\Delta z=-0.135 \AA$, and red corresponding to $\Delta z=0.345 \AA$. The characteristic inter-plane distance for $z$ is $d_{h k l}=d_{\overline{1} 10}=2.240 \AA$. Visualisation was prepared using the Ovito [89] program. 
ratio of $A=0.72$ [91], compared to $A=1$ for an elastic isotropic material. While analytical models suitable for anisotropic materials are available, their involvement makes comparisons notably more cumbersome. Difficulties associated with anisotropic models were highlighted e.g. in [92], and, more recently e.g. in [71]. Since this work is devoted mainly to the presentation of the relevant CFD expressions, we postpone in-depth analysis employing more complex models for a future communication.

Even a brief visual examination of figures 4 and 5 reveals that the results of atomistic simulations are in very good agreement with theoretical predictions. For each of the investigated components $\sigma_{x x}, \sigma_{y y}, \sigma_{z z}$, and $\sigma_{x z}$ the curves obtained from linear elasticity theory are consistent with the predictions of Hardy stress, particularly further from the dislocation line, i.e. for $|x|$ and $|z|>5 b$. In the vicinity of the dislocation line discrepancies become evident, which is most clearly seen for the crosssection $x=0 b$ and components $\sigma_{x x}, \sigma_{y y}$, and $\sigma_{z z}$ (figure 4, panels a-c, red curve), where the predicitions no longer agree. Other notable disagreements are apparent for $\sigma_{x z}$ and cross-sections $z=0 b, 7.5 b, 15 b$ (figure 5, panel d, all curves). These differences should not be construed to result from deficiencies of the atomistic model employed here, but rather as indication of the breakdown of linear elasticity theory, which at close range becomes unphysical, as evidenced by the singularities in its predictions. This is, of course, expected close to the dislocation core - a regime, where nonlinearity becomes significant. The atomistic approach, owing to its explicit taking into account the discrete nature of matter, along with an accurate s-MEAM description of individual interatomic interactions, offers a much more realistic description of the stress distribution in this regime - a clear advantage of atomistic techniques over
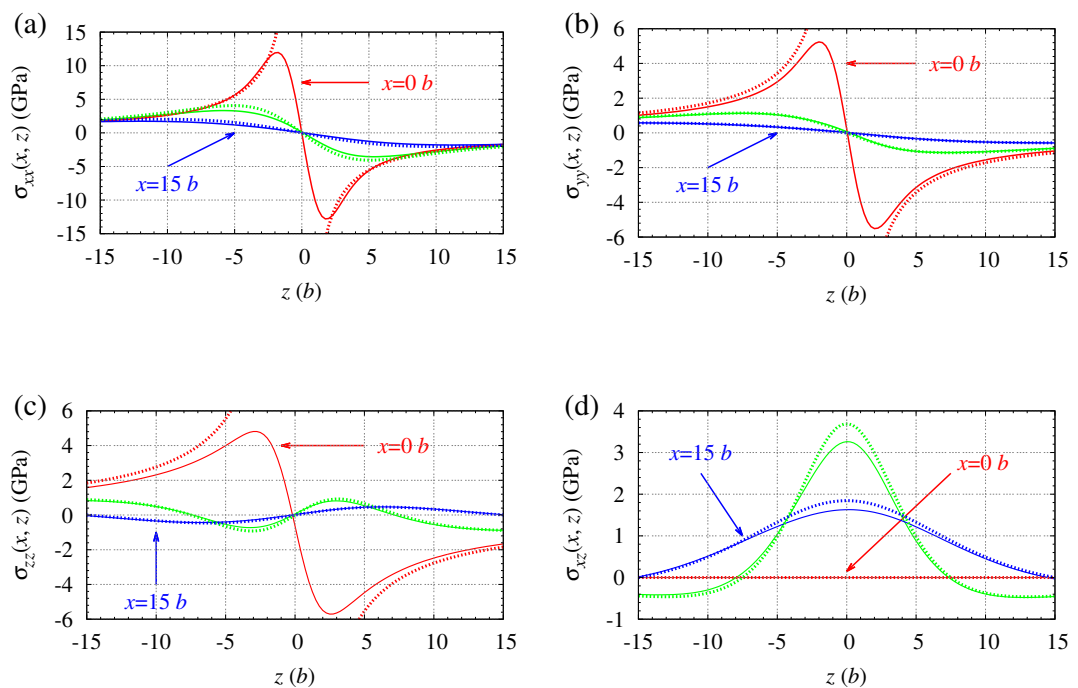

Figure 4. Stress distribution due to an edge dislocation with a Burgers vector $\mathbf{b}=1 / 2\langle 111\rangle$ in a bcc monocrystal of Mo. Subsequent panels compare the results of atomistic calculations (solid line) with the predictions of linear elasticity theory (dotted lines) for the components $\sigma_{x x}, \sigma_{y y}, \sigma_{z z}$, and $\sigma_{x z}$ of the stress tensor on cross-sections $x=0 b$ (red), $x=7.5 b$ (green), and $x=15 b$ (blue). The theoretical predictions assume that the material is isotropic. 

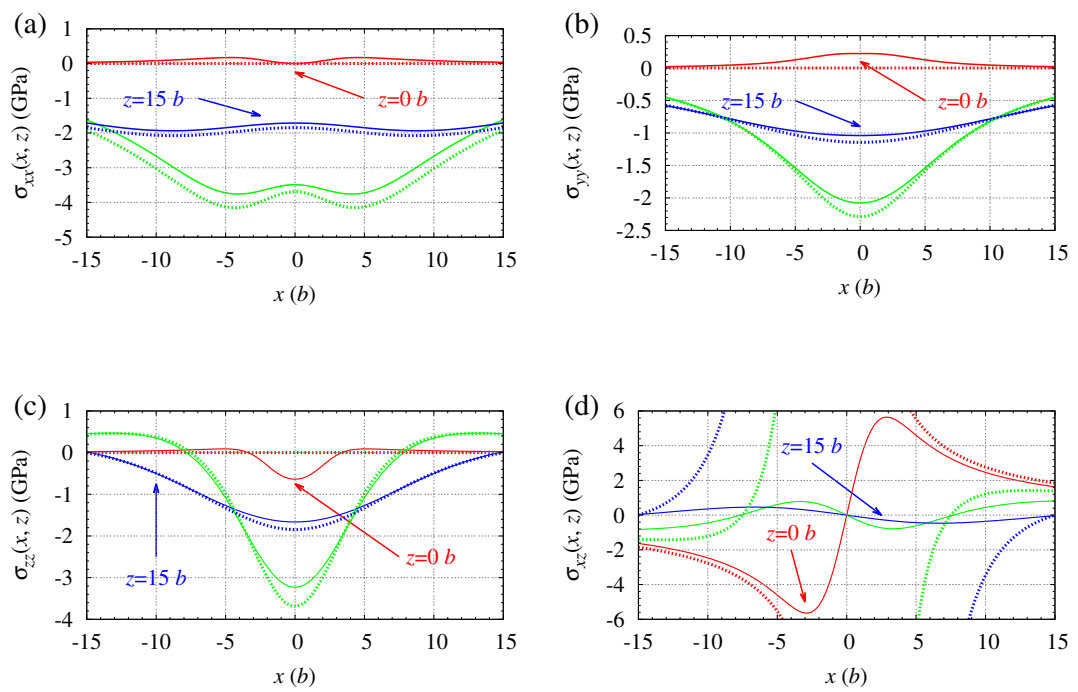

Figure 5. Stress distribution due to an edge dislocation with a Burgers vector $\mathbf{b}=1 / 2\langle 111\rangle$ in a bcc monocrystal of Mo. Subsequent panels compare the results of atomistic calculations (solid line) with the predictions of linear elasticity theory (dotted lines) for the components $\sigma_{x x}, \sigma_{y y}, \sigma_{z z}$, and $\sigma_{x z}$ of the stress tensor on cross-sections $z=0 b$ (red), $z=7.5 b$ (green), and $z=15 b$ (blue). The theoretical predictions assume that the material is isotropic.

continuum methods. This concludes our demonstration of the viability of the proposed CFD expressions for the s-MEAM class of potentials.

\section{Summary and final remarks}

In this work we derived a central-force decomposition for potentials of the s-MEAM type. We followed the derivation with a brief discussion of the decomposition, demonstrating that the s-MEAM potential is characterised by non-vanishing central interactions between not only first-nearest, but also second-nearest neighbours. We applied the derived CFD in a brief study of stress fields due to an edge dislocation in bcc molybdenum, demonstrating the practicability of our approach and good agreement with the predictions of linear elasticity theory in the region away from the dislocation core, and the superiority of the atomistic approach in the immediate vicinity of the dislocation core, where the continuum description breaks down.

The main outcome of this work are the expressions (52)-(53), which, despite being a result of careful simplifications, remain computationally expensive. This is particularly the case for the quantity $\Theta_{i j}$ defined by (53), whose evaluation necessitates calculating products of $\cos \theta$, the functions $f(r)$ and $g(\cos \theta)$, and their derivatives. The computational effort associated with this term, calculated as is, is expected to be significant. Building on the theoretical foundation of this work, in a future paper we expect to describe a pragmatic, computationally efficient technique for the evaluation of the CFD derived here.

The fact that the calculation of Hardy stress for an atomic systems necessitates 
performing a central-force decomposition for the potential of choice was the prime motivation of this work. However, we feel obliged to point out a number of computational techniques that will also benefit from the presented CFD.

A central-force decomposition provides an expression for the first derivative of a potential energy with respect to interatomic distance, i.e. $\partial E_{\text {pot }} / \partial r_{\alpha \beta}$. Being able to calculate this quantity is a necessary condition for applying the formalism proposed by Ray et al [93] to the study of mechanical properties of atomic systems. This formalism allows the determination of elastic constants (both adiabatic, and isothermal) of a general anisotropic material. The use of this approach necessitates being able to calculate not only the above-mentioned first derivative, but also $\partial^{2} E_{\text {pot }} / \partial r_{\alpha \beta} \partial r_{\eta \xi}$. In this context, the results presented here can be seen as a stepping stone towards the use of s-MEAM potentials to study the mechanical properties of matter (such as stress distributions or elastic constants), with the obvious requirement of deriving the expressions for the second derivative next.

The expressions for both of the above-mentioned derivatives are also of use in recently-proposed thermostats based on the concept of configurational temperature [94-96]. The results presented here can thus also be viewed as facilitating future studies of non-equilibrium properties, for which such thermostats are invaluable.

Finally, the presented central-force decomposition constitutes a novel interpretation tool. The significant formal complexity of the s-MEAM potential makes it difficult to assess how the features of the individual functions defining the potential affect the resultant (effective) interactions, and, in consequence, how they influence the dynamics and properties of the modeled system. The obtained CFD, by virtue of expressing a complicated many-body picture in a language of easy-to-interpret, central, pairwise interactions significantly facilitates the understanding of the nature of bonding in systems under study. We hope to present applications of this CFD as an interpretation tool in the near future.

\section{Acknowledgments}

We acknowledge the support of the Polish Ministry of Science and Higher Education (grant IP2012 043972) and of the TASK Academic Computer Centre (Gdańsk, Poland). This research was also supported in part by the PL-Grid Infrastructure (grant POIG.02.03.00-00-096/10).

\section{References}

[1] D G Pettifor and I I Oleinik. Analytic bond-order potentials beyond Tersoff-Brenner. I. Theory. Phys. Rev. B, 59:8487-8499, Apr 1999.

[2] D G Pettifor and I I Oleinik. Bounded analytic bond-order potentials for $\sigma$ and $\pi$ bonds. Phys. Rev. Lett., 84:4124-4127, May 2000.

[3] D G Pettifor and I I Oleinik. Analytic bond-order potential for open and close-packed phases. Phys. Rev. B, 65:172103, Apr 2002.

[4] D G Pettifor, M W Finnis, D Nguyen-Manh, D A Murdick, X W Zhou, and H N G Wadley. Analytic bond-order potentials for multicomponent systems. Materials Science and Engineering: A, 365(1-2):2-13, 2004. Multiscale Materials Modelling.

[5] R Drautz, D A Murdick, D Nguyen-Manh, X Zhou, H N G Wadley, and D G Pettifor. Analytic bond-order potential for predicting structural trends across the sp-valent elements. Phys. Rev. B, 72:144105, Oct 2005

[6] D A Murdick, X W Zhou, and H N G Wadley. Assessment of interatomic potentials for molecular dynamics simulations of GaAs deposition. Phys. Rev. B, 72:205340, Nov 2005. 
[7] D A Murdick, X W Zhou, H N G Wadley, D Nguyen-Manh, R Drautz, and D G Pettifor. Analytic bond-order potential for the gallium arsenide system. Phys. Rev. B, 73:045206, Jan 2006.

[8] K Albe, K Nordlund, J Nord, and A Kuronen. Modeling of compound semiconductors: Analytical bond-order potential for Ga, As, and GaAs. Phys. Rev. B, 66:035205, Jul 2002.

[9] S J Stuart, A B Tutein, and J A Harrison. A reactive potential for hydrocarbons with intermolecular interactions. The Journal of Chemical Physics, 112(14):6472-6486, 2000.

[10] D W Brenner, O A Shenderova, J A Harrison, S J Stuart, B Ni, and S B Sinnott. A second-generation reactive empirical bond order (REBO) potential energy expression for hydrocarbons. Journal of Physics: Condensed Matter, 14(4):783, 2002.

[11] T J Lenosky, B Sadigh, E Alonso, V V Bulatov, T D de la Rubia, J Kim, A F Voter, and J D Kress. Highly optimized empirical potential model of silicon. Modelling and Simulation in Materials Science and Engineering, 8(6):825, 2000.

[12] D K Ward, X W Zhou, B M Wong, F P Doty, and J A Zimmerman. Analytical bond-order potential for the cadmium telluride binary system. Phys. Rev. B, 85:115206, Mar 2012.

[13] D K Ward, X W Zhou, B M Wong, F P Doty, and J A Zimmerman. Analytical bond-order potential for the Cd-Zn-Te ternary system. Phys. Rev. B, 86:245203, Dec 2012.

[14] F Hutchinson, M Wilson, and P A Madden. Short- and intermediate-range order in molten metal tribromides: a computer simulation study. Journal of Physics: Condensed Matter, 12(50):10389, 2000.

[15] X W Zhou and F P Doty. Embedded-ion method: An analytical energy-conserving chargetransfer interatomic potential and its application to the La-Br system. Phys. Rev. B, 78:224307, Dec 2008.

[16] Y Mishin, D Farkas, M J Mehl, and D A Papaconstantopoulos. Interatomic potentials for monoatomic metals from experimental data and ab initio calculations. Phys. Rev. B, 59:33933407, Feb 1999.

[17] B-J Lee and M I Baskes. Second nearest-neighbor modified embedded-atom-method potential. Phys. Rev. B, 62:8564-8567, Oct 2000.

[18] X D Dai, Y Kong, J H Li, and B X Liu. Extended Finnis-Sinclair potential for bcc and fcc metals and alloys. Journal of Physics: Condensed Matter, 18(19):4527, 2006.

[19] X W Zhou, J A Zimmerman, B M Wong, and J J Hoyt. An embedded-atom method interatomic potential for Pd-H alloys. Journal of Materials Research, 23:704-718, 2008.

[20] A M Dongare, M Neurock, and L V Zhigilei. Angular-dependent embedded atom method potential for atomistic simulations of metal-covalent systems. Phys. Rev. B, 80:184106, Nov 2009.

[21] H W Sheng, M J Kramer, A Cadien, T Fujita, and M W Chen. Highly optimized embeddedatom-method potentials for fourteen fcc metals. Phys. Rev. B, 83:134118, Apr 2011.

[22] H Park, M R Fellinger, T J Lenosky, W W Tipton, D R Trinkle, S P Rudin, C Woodward, J W Wilkins, and R G Hennig. Ab initio based empirical potential used to study the mechanical properties of molybdenum. Phys. Rev. B, 85:214121, Jun 2012.

[23] M S Daw and M I Baskes. Embedded-atom method: Derivation and application to impurities, surfaces, and other defects in metals. Phys. Rev. B, 29:6443-6453, Jun 1984.

[24] A P Sutton and J Chen. Long-range Finnis-Sinclair potentials. Philosophical Magazine Letters, 61(3):139-146, 1990

[25] M S Daw, S M Foiles, and M I Baskes. The embedded-atom method: a review of theory and applications. Materials Science Reports, 9(7-8):251-310, 1993.

[26] M I Baskes. Application of the embedded-atom method to covalent materials: A semiempirical potential for silicon. Phys. Rev. Lett., 59:2666-2669, Dec 1987.

[27] M I Baskes. Modified embedded-atom potentials for cubic materials and impurities. Phys. Rev. B, 46:2727-2742, Aug 1992.

[28] Y-M Kim, B-J Lee, and M I Baskes. Modified embedded-atom method interatomic potentials for Ti and Zr. Phys. Rev. B, 74:014101, Jul 2006.

[29] F Apostol and Y Mishin. Interatomic potential for the Al-Cu system. Phys. Rev. B, 83:054116, Feb 2011.

[30] S Winczewski. Algorithms for locating dislocations in numerically simulated materials. PhD thesis, Gdansk University of Technology, 2015.

[31] R G Hennig, T J Lenosky, D R Trinkle, S P Rudin, and J W Wilkins. Classical potential describes martensitic phase transformations between the $\alpha, \beta$, and $\omega$ titanium phases. Phys. Rev. B, 78:054121, Aug 2008.

[32] Y A Du, T J Lenosky, R G Hennig, S Goedecker, and J W Wilkins. Energy landscape of silicon tetra-interstitials using an optimized classical potential. physica status solidi (b), 
248(9):2050-2055, 2011.

[33] R Clausius. XVI. On a mechanical theorem applicable to heat. Philosophical Magazine Series 4, 40:122-127, 1870.

[34] J C Maxwell. On reciprocal figures, frames and diagrams of forces. Trans. R. Soc. Edinburgh, XXVI:1-43, 1870

[35] J C Maxwell. van der Waals on the continuity of the gaseous and liquid states. Nature (London), 10:477-480, 1874.

[36] D H Tsai. The virial theorem and stress calculation in molecular dynamics. The Journal of Chemical Physics, 70(3):1375-1382, 1979.

[37] Z S Basinski, M S Duesbery, and R Taylor. Can. J. Phys, 49:2160, 1971.

[38] J F Lutsko. Stress and elastic constants in anisotropic solids: Molecular dynamics techniques. Journal of Applied Physics, 64(3):1152-1154, 1988.

[39] M Zhou. A new look at the atomic level virial stress: on continuum-molecular system equivalence. Proceedings of the Royal Society of London A: Mathematical, Physical and Engineering Sciences, 459(2037):2347-2392, 2003.

[40] J Cormier, J M Rickman, and T J Delph. Stress calculation in atomistic simulations of perfect and imperfect solids. Journal of Applied Physics, 89(1):99-104, 2001.

[41] J H Irving and J G Kirkwood. The Statistical Mechanical Theory of Transport Processes. IV. The Equations of Hydrodynamics. The Journal of Chemical Physics, 18(6):817-829, 1950.

[42] W Noll. Die Herleitung der Grundgleichungen der Thermomechanik der Kontinua aus der Statistichen Mechanik. Journal of Rational Mechanics and Analysis, 4:627-646, 1955.

[43] R J Hardy. Formulas for determining local properties in molecular-dynamics simulations: Shock waves. The Journal of Chemical Physics, 76(1):622-628, 1982.

[44] A I Murdoch. The motivation of continuum concepts and relations from discrete considerations. Q. J. Mech. Appl. Math., 36:163-187, 1983.

[45] A I Murdoch and D Bedeaux. On the physical interpretation of fields in continuum mechanics. International Journal of Engineering Science, 31(10):1345 - 1373, 1993.

[46] A I Murdoch and D Bedeaux. Continuum equations of balance via weighted averages of microscopic quantities. Proceedings of the Royal Society of London A: Mathematical, Physical and Engineering Sciences, 445(1923):157-179, 1994.

[47] S Root, R J Hardy, and D R Swanson. Continuum predictions from molecular dynamics simulations: shock waves. The Journal of Chemical Physics, 118(7):3161-3165, 2003.

[48] A I Murdoch. A critique of atomistic definitions of the stress tensor. Journal of Elasticity, $88(2): 113-140,2007$.

[49] A I Murdoch. On molecular modelling and continuum concepts. Journal of Elasticity, 100(12):33-61, 2010.

[50] S Shen and S N Atluri. Atomic-level stress calculation and continuum-molecular system equivalence. Computer Modelling in Engineering and Sciences, 6:91, 2004.

[51] E B Webb III, J A Zimmerman, and S C Seel. Reconsideration of continuum thermomechanical quantities in atomic scale simulations. Mathematics and Mechanics of Solids, 13(3-4):221$266,2008$.

[52] J Z Yang, X Wu, and X Li. A generalized Irving-Kirkwood formula for the calculation of stress in molecular dynamics models. The Journal of Chemical Physics, 137(13):134104, 2012.

[53] N C Admal and E B Tadmor. A unified interpretation of stress in molecular systems. Journal of Elasticity, 100(1-2):63-143, 2010.

[54] N C Admal and E B Tadmor. Stress and heat flux for arbitrary multibody potentials: A unified framework. The Journal of Chemical Physics, 134(18):184106, 2011.

[55] J A Zimmerman, E B Webb III, J J Hoyt, R E Jones, P A Klein, and D J Bammann. Calculation of stress in atomistic simulation. Modelling and Simulation in Materials Science and Engineering, 12(4):S319, 2004.

[56] Y Chen. Local stress and heat flux in atomistic systems involving three-body forces. The Journal of Chemical Physics, 124(5):-, 2006.

[57] J A Zimmerman, R E Jones, and J A Templeton. A material frame approach for evaluating continuum variables in atomistic simulations. Journal of Computational Physics, 229(6):2364 $-2389,2010$

[58] R E Jones, J A Zimmerman, J Oswald, and T Belytschko. An atomistic j-integral at finite temperature based on hardy estimates of continuum fields. Journal of Physics: Condensed Matter, 23(1):015002, 2011.

[59] M H Ulz, K K Mandadapu, and P Papadopoulos. On the estimation of spatial averaging volume for determining stress using atomistic methods. Modelling and Simulation in Materials Science and Engineering, 21(1):015010, 2013. 
[60] Y Fu and A C To. On the evaluation of hardy's thermomechanical quantities using ensemble and time averaging. Modelling and Simulation in Materials Science and Engineering, 21(5):055015, 2013.

[61] Y Fu and A C To. A modification to Hardy's thermomechanical theory that conserves fundamental properties more accurately. Journal of Applied Physics, 113(23):233505, 2013.

[62] Y Fu and A C To. A modification to Hardy's thermomechanical theory for conserving fundamental properties more accurately: tensile and shear failures in iron. Modelling and Simulation in Materials Science and Engineering, 22(1):015010, 2014.

[63] Y Fu and J-H Song. On computing stress in polymer systems involving multi-body potentials from molecular dynamics simulation. The Journal of Chemical Physics, 141:054108, 2014.

[64] J M Vanegas, A Torres-Sánchez, and M Arroyo. Importance of force decomposition for local stress calculations in biomembrane molecular simulations. Journal of Chemical Theory and Computation, 10(2):691-702, 2014.

[65] S Morante, G C Rossi, and M Testa. The stress tensor of a molecular system: An exercise in statistical mechanics. The Journal of Chemical Physics, 125(3):-, 2006.

[66] F H Stillinger and T A Weber. Computer simulation of local order in condensed phases of silicon. Phys. Rev. B, 31:5262-5271, Apr 1985.

[67] S Goedecker. Optimization and parallelization of a force field for silicon using OpenMP. Computer Physics Communications, 148(1):124 - 135, 2002.

[68] S Plimpton. Fast parallel algorithms for short-range molecular dynamics. Journal of Computational Physics, 117(1):1 - 19, 1995.

[69] I Kovács and L Zsoldos. Appendix C - The stress-fields of straight edge and screw dislocations. In Dislocations and Plastic Deformation, volume 60 of International Series in Natural Philosophy, pages 320 - 325. Pergamon, 1973.

[70] D Hull and D J Bacon. Chapter 4 - elastic properties of dislocations. In Introduction to Dislocations, pages 63 - 83. Butterworth-Heinemann, Oxford, fifth edition, 2011.

[71] D Seif, G Po, M Mrovec, M Lazar, C Elsässer, and P Gumbsch. Atomistically enabled nonsingular anisotropic elastic representation of near-core dislocation stress fields in $\alpha$-iron. Phys. Rev. B, 91:184102, May 2015.

[72] V V Bulatov and W Cai. Computer Simulations of Dislocations. Oxford University Press, 2006.

[73] E B Tadmor and R E Miller. Molecular statics. In Modeling Materials, pages 304-374. Cambridge University Press, 2011. Cambridge Books Online.

[74] Y N Osetsky and D J Bacon. An atomic-level model for studying the dynamics of edge dislocations in metals. Modelling and Simulation in Materials Science and Engineering, $11(4): 427,2003$.

[75] M I Baskes and M S Daw. 4th Int. Conf. on the Effects of Hydrogen on the Behaviour of Materials (jackson lake lodge, moran, wy). In $\mathrm{N}$ Moody and A Thompson, editors, 4th Int. Conf. on the Effects of Hydrogen on the Behaviour of Materials (Jackson Lake Lodge, Moran, WY), 1989. Warrendale, PA: The Minerals, Metals and Materials Society.

[76] J Chang, W Cai, V V Bulatov, and S Yip. Dislocation motion in BCC metals by molecular dynamics. Materials Science and Engineering: A, 309-310:160, 2001.

[77] J Chang, W Cai, V V Bulatov, and S Yip. Molecular dynamics simulations of motion of edge and screw dislocations in a metal. Computational Materials Science, 23:111, 2002.

[78] G Monnet. Mechanical and energetical analysis of molecular dynamics simulations of dislocationdefect interactions. Acta Materialia, 55:5081, 2007.

[79] A Y Kuksin, V V Stegailov, and A V Yanilkin. Molecular dynamics simulation of edgedislocation dynamics in aluminium. Doklady Physics, 53:287, 2008.

[80] G Monnet and D Terentyev. Structure and mobility of the $1 / 2\langle 111\rangle\{112\}$ edge dislocation in BCC iron studied by molecular dynamics. Acta Materialia, 57(5):1416, 2009.

[81] D J Bacon, Y N Osetsky, and D Rodney. Chapter 88 Dislocation-Obstacle Interactions at the Atomic Level. In J. P. Hirth and L. Kubin, editors, Dislocations in Solids, volume 15, page 1. Elsevier, 2009.

[82] M R Gilbert, S Queyreau, and J Marian. Stress and temperature dependence of screw dislocation mobility in $\alpha$-Fe by molecular dynamics. Phys. Rev. B, 84:174103, 2011.

[83] S Queyreau, J Marian, M R Gilbert, and B D Wirth. Edge dislocation mobilities in bcc Fe obtained by molecular dynamics. Phys. Rev. B, 84:064106, 2011.

[84] H-X Xie, L Bo, and T Yu. Molecular dynamics simulation of an edge dislocation slipping on a cubic plane of Ni 3 Al. Modelling and Simulation in Materials Science and Engineering, 19:065005, 2011.

[85] D Cereceda, J M Perlado, and J Marian. Techniques to accelerate convergence of stress- 
controlled molecular dynamics simulations of dislocation motion. Comp. Mat. Sci., 62:272, 2012.

[86] K Kang, V V Bulatov, and W Cai. Singular orientations and faceted motion of dislocations in body-centered cubic crystals. Proceedings of the National Academy of Sciences, 109(38):15174, 2012

[87] E Polak and G Ribiére. Note sur la convergence de méthodes de directions conjuguées. ESAIM: Mathematical Modelling and Numerical Analysis - Modélisation Mathématique et Analyse Numérique, 3(R1):35-43, 1969.

[88] L Dagum and R Enon. OpenMP: an industry standard API for shared-memory programming. Computational Science \& Engineering, IEEE, 5(1):46-55, 1998.

[89] A Stukowski. Visualization and analysis of atomistic simulation data with OVITO-the Open Visualization Tool. Modelling and Simulation in Materials Science and Engineering, 18(1):015012, 2010.

[90] G V Samsonov, editor. Handbook of the Physicochemical Properties of the Elements, chapter Mechanical Properties of the Elements, pages 387-446. Springer US, Boston, MA, 1968.

[91] T S Duffy, G Shen, J Shu, H-K Mao, R J Hemley, and A K Singh. Elasticity, shear strength, and equation of state of molybdenum and gold from x-ray diffraction under nonhydrostatic compression to 24 gpa. Journal of Applied Physics, 86(12):6729-6736, 1999.

[92] J Lothe. Chapter 4 - Dislocations in Anisotropic Media. In V L Indenbom and J Lothe, editors, Elastic Strain Fields and Dislocation Mobility, volume 31 of Modern Problems in Condensed Matter Sciences, pages 269 - 328. Elsevier, 1992.

[93] J R Ray and A Rahman. Statistical ensembles and molecular dynamics studies of anisotropic solids. The Journal of Chemical Physics, 80(9):4423-4428, 1984.

[94] J Delhommelle and D J Evans. Configurational temperature thermostat for fluids undergoing shear flow: application to liquid chlorine. Molecular Physics, 99(21):1825-1829, 2001.

[95] C Braga and K P Travis. A configurational temperature Nosé-Hoover thermostat. The Journal of Chemical Physics, 123(13):134101, 2005.

[96] S Pieprzyk, D M Heyes, S Maćkowiak, and A C Brańka. Galilean-invariant Nosé-Hoover-type thermostats. Phys. Rev. E, 91:033312, Mar 2015. 